\title{
Electrical Impedance tomography
}

Adler, Andy and Gaburro, Romina and Lionheart, William

2015

MIMS EPrint: 2020.23

Manchester Institute for Mathematical Sciences

School of Mathematics

The University of Manchester

\footnotetext{
Reports available from: http://eprints.maths.manchester.ac.uk/

And by contacting: The MIMS Secretary

School of Mathematics

The University of Manchester

Manchester, M13 9PL, UK
} 


\title{
Excerpt from: Handbook of Mathematical Methods in Imaging, edited by Otmar Scherzer.
}

\author{
Cite as: A. Adler, R. Gaburro, and W. Lionheart. "Electrical impedance \\ tomography". Handbook of Mathematical Methods in Imaging ed O Scherzer." 2nd \\ edition (2015): 701-762. We encourage the reader to consult the published version of \\ this chapter if possible, and the book that contains it. These are online via Springer \\ Link if your institution has a subscription. The published version has links to other \\ chapters in the book and of course pages are numbered differently from this preprint
}




\section{Electrical Impedance Tomography}

Andy Adler, Romina Gaburro, William Lionheart 


\section{$0.1 \quad$ Introduction}

Electrical Impedance Tomography (EIT) is the recovery of the conductivity (or conductivity and permittivity) of the interior of a body from a knowledge of currents and voltages applied to its surface. In geophysics, where the method is used in prospecting and archaeology, it is known as electrical resistivity tomography. In industrial process tomography it is known as electrical resistance tomography or electrical capacitance tomography. In medical imaging, when at the time of writing it is still an experimental technique rather than routine clinical practice, it is called EIT. A very similar technique is used by weakly electric fish to navigate and locate prey and in this context it is called electrosensing. An example of a medical application of EIT is given in Figure 1, which shows a ten day old healthy neonate breathing spontaneously and lying in the prone position with the head turned to the left. Sixteen EIT electrodes are placed in a transverse plane around the chest, and EIT data acquired with the Goe MF-II system. This child was a subject in a study which used EIT to examine patterns of breathing in neonates and the relationship to body posture [59]. In this study, EIT was able to show, for the first time, that, in a prone position, the lung on the opposite side (contralateral) to the face receives significantly larger air flows.

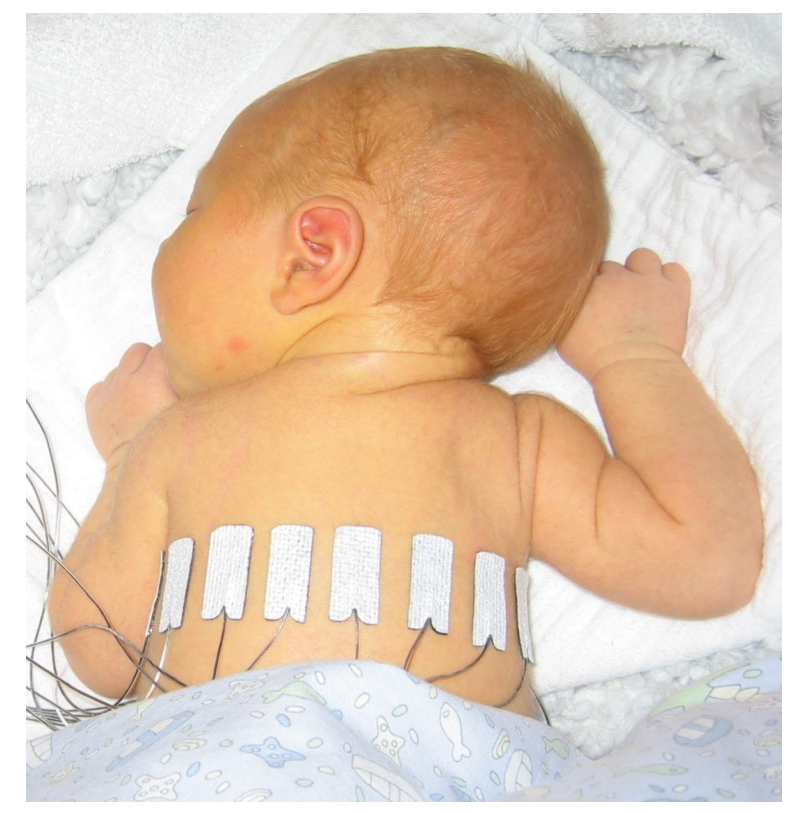

Figure 1: 10-day old spontaneously breathing neonate lying in the prone position with the head turned to the left. Sixteen medical grade $\mathrm{Ag} / \mathrm{AgCl}$ electrodes were placed in a transverse plane and connected to a Geo MF-II EIT system[59]

The simplest mathematical formulation of inverse problem of EIT can be 
stated as follows. Let $\Omega$ be a conducting body described by a bounded domain in $\mathbb{R}^{n}, n \geq 2$, with electrical conductivity a bounded and positive function $\gamma(x)$ (later we will consider also $\gamma$ complex). In absence of internal sources, the electrostatic potential $u$ in $\Omega$, is governed by the elliptic partial differential equation

$$
L_{\gamma} u:=\nabla \cdot \gamma \nabla u=0 \quad \text { in } \quad \Omega .
$$

It is natural to consider the weak formulation of 0.1 .1 in which $u \in H^{1}(\Omega)$ is a weak solution to (0.1.1). Given a potential $\phi \in H^{1 / 2}(\partial \Omega)$ on the boundary, the induced potential $u \in H^{1}(\Omega)$ solves the Dirichlet problem

$$
\left\{\begin{array}{l}
L_{\gamma} u=0 \quad \text { in } \quad \Omega \\
\left.u\right|_{\partial \Omega}=\phi .
\end{array}\right.
$$

The currents and voltages measurements taken on the surface of $\Omega, \partial \Omega$, are given by the so-called Dirichlet-to-Neumann map (associated with $\gamma$ ) or voltage-to-current map

$$
\Lambda_{\gamma}:\left.u\right|_{\partial \Omega} \in H^{1 / 2}(\partial \Omega) \longrightarrow \gamma \frac{\partial u}{\partial \nu} \in H^{-1 / 2}(\partial \Omega) .
$$

Here, $\nu$ denotes the unit outer normal to $\partial \Omega$ and the restriction to the boundary is considered in the sense of the trace theorem on Sobolev spaces. We require that $\partial \Omega$ be at least Lipschitz continuous and $\gamma \in L^{\infty}(\Omega)$ with ess inf $\operatorname{Re} \gamma=m>0$.

The forward problem under consideration is the map $\gamma \in \mathcal{D}_{m} \mapsto \Lambda_{\gamma}$, where $\mathcal{D}_{m}=\left\{\gamma \in L^{\infty}(\Omega) \mid\right.$ ess $\left.\inf \gamma \geq m\right\}$ The inverse problem for complete data is then the recovery of $\gamma$ from $\Lambda_{\gamma}$. As is usual in inverse problems we will consider the questions of (1) uniqueness of solution (or from a practical point of view sufficiency of data) (2) stability/instability with respect to errors in the data and (3) practical algorithms for reconstruction. It is also worth pointing out to the reader who is not very familiar with EIT the well known fact that the behavior of materials under the influence of external electric fields is determined not only by the electrical conductivity $\gamma$ but also by the electric permittivity $\varepsilon$ so that the determination of the complex valued function $\gamma(x, \omega)=\sigma(x)+\mathrm{i} \omega \varepsilon(x)$, would be the more general and realistic problem, where $\mathrm{i}=\sqrt{-1}$ and $\omega$ is the frequency. The simple case where $\omega=0$ will be treated in this work. For a description of the formulation of the inverse problem for the complex case we refer for example to [17]. Before we address questions (1)-(3) mentioned above, we will consider how the problem arises in practice.

\subsubsection{Measurement systems and physical derivation}

For the case of direct current, that is the voltage applied is independent of time, the derivation is simple. Of course here $\Omega \subset \mathbb{R}^{3}$. Let us first suppose that we can apply an arbitrary voltage $\phi \in H^{1 / 2}(\Omega)$ to the surface. We assume that the exterior $\mathbb{R}^{3} \backslash \Omega$ is an electrical insulator. An electric potential (voltage) $u$ 
results in the interior and the current $\mathbf{J}$ that flows satisfies the continuum Ohm's law $\mathbf{J}=-\gamma \nabla u$; the absence of current sources in the interior is expressed by the continuum version of Kirchoff's law $\nabla \cdot \mathbf{J}=0$ which together result in 0.1.1. The boundary conditions are controlled or measured using a system of conducting electrodes which are typically applied to the surface of the object. In some applications, especially geophysical, these may be spikes that penetrate the object, but it is common to model these as points on the surface. Systems are used that to a reasonable approximation apply a known current on (possibly a subset) or electrodes and measure the voltage that results on electrodes (again possibly a subset, in some cases disjoint from those carrying a non-zero current). In other cases it is a predetermined voltage applied to electrodes and the current measured; there being practical reasons determined by electronics or safety for choosing one over the other. In medical EIT applying known currents and measuring voltages is typical. One reason for this is the desire to limit the maximum current for safety reasons. In practice the circuit that delivers a predetermined current can only do so while the voltage required to do that is within a certain range so both maximum current and voltage are limited. For an electrode (let us say indexed by $\ell$ ) not modelled by a point but covering a region $E_{\ell} \subset \partial \Omega$ the current to that electrode is the integral

$$
I_{\ell}=\int_{E_{\ell}}-\mathbf{J} \cdot \nu d x .
$$

Away from electrodes we have

$$
\gamma \frac{\partial u}{\partial \nu}=0, \quad \text { on } \partial \Omega \backslash \bigcup_{\ell=1}^{L} E_{\ell}
$$

as the air surrounding the object is an insulator. On the conducting electrode we have $\left.u\right|_{E_{\ell}}=V_{\ell}$ a constant, or as a differential condition

$$
\nu \times \nabla u=0 \quad \text { on } \partial \Omega \backslash \bigcup_{\ell=1}^{L} E_{\ell} .
$$

Taken together, (0.1.3)-(0.1.5) are called the shunt model. This ideal of a perfectly conducting electrode is of course only an approximation, and we note that while the condition $u \in H^{1}(\Omega)$ is a sensible condition, ensuring finite total dissipated power, it is not sufficient to ensure (0.1.3) is well defined. Indeed for smooth $\gamma$ and smooth $\partial E_{\ell}$ the condition results in a square root singularity in the current density on the electrode. We will come back to a more realistic model of electrodes.

It is more common to use alternating current in geophysical and process monitoring applications, and essential in medical applications. Specifically the direction of the current must be reversed within a sufficiently short time to avoid electrochemical effects. This also means that the time average of the applied current should be zero. In medical applications, current in one direction for 
sufficient duration would result in transport of ions, and one of the effects of this can be stimulation of nerves. It would also degrade electrode behavior due to charge build up and ionic changes in the electrode. As a general rule higher levels of current and voltage are considered safer at higher temporal frequencies. The simplest EIT system therefore operates at a fixed frequency using an oscillator or digital signal processing to produce a sinusoidal current. Measurements are then taken of the magnitude, or in the some cases the components that are in phase and $\pi / 2$ out of phase with the original sine wave. Of course when current or voltage is first applied to the object a transient results, and typical EIT systems are designed to start measuring after this transient term has decayed so as to be negligible.

In geophysics a technique that is complementary to EIT called induced polarization tomography IPT is used to find polarizable minerals. In effect this uses a square wave pulse and measures the transient response [86]. In process tomography a technique known as electrical capacitance tomography is designed for imaging insulating materials with different dielectric permittivities, for example oil and gas in a pipe[56] [103]. Again square waves or pulses are used.

In medical and geophysical problems the response of the materials may vary with frequency. For example in a biological cell higher frequency current might penetrate a largely capacitive membrane and so be influence by the internal structures of the cell while lower frequency currents pass around the cell. This has led to Electrical Impedance Tomography Spectroscopy (EITS)[48], and in geophysics Spectral Induced Polarization Tomography (SIPT)[86]. The spectral response can be established either by using multiple sinusoidal frequencies or by sampling the transient response to a pulse.

Our starting point for the case of alternating current is the time harmonic Maxwell equations at a fixed angular frequency $\omega$. Here it is assumed that the transient components of all fields are negligible and represent the time harmonic electric and magnetics vector fields using the complex representation $\mathcal{F}(x, t)=$ $\operatorname{Re}(\mathbf{F} \exp (\mathrm{i} \omega t))$ and we have

$$
\begin{aligned}
\nabla \times \mathbf{E} & =-\mathrm{i} \omega \mathbf{B} \\
\nabla \times \mathbf{H} & =\mathbf{J}+\mathrm{i} \omega \mathbf{D} .
\end{aligned}
$$

The electric and magnetic fields $\mathbf{E}$ and $\mathbf{H}$ are related to the current density $\mathbf{J}$, electric displacement $\mathbf{D}$ and magnetic flux $\mathbf{B}$ by the material properties conductivity $\sigma$, permittivity $\epsilon$ and permeability $\mu$ by

$$
\mathbf{J}=\sigma \mathbf{E}, \mathbf{D}=\epsilon \mathbf{E}, \mathbf{B}=\mu \mathbf{H} .
$$

The fields $\mathbf{E}$ and $\mathbf{H}$ are evaluated on directed curves, while the "fluxes" J, D and $\mathbf{B}$ on surfaces. In biomedical applications one can typically take $\mu$ to be constant and to be the same inside the body as outside in air. In non-destructive testing and geophysical applications there may well be materials with differing permeability. We are also assuming linear relations in (0.1.8). For example the 
first is the continuum Ohm's law. We allow for the possibility that the material properties are frequency dependent. In this, dispersion is important in EIS and SIPT. For the moment we also assume isotropy (so that the material properties are scalars).

There are many inverse problems governed by time harmonic Maxwell's equations. For very large values of $\omega$ this includes optical and microwave tomographic techniques and scattering problems such as radar which we do not discus in this chapter. There are also systems where the fields arise from alternating current in a coil, and measurements are made either with electrodes or with other coils. Mutual (or magnetic) induction tomography (MIT) falls in to this category and has been tried in medical and process monitoring applications [49]. In these cases the eddy current approximation [9] to Maxwell's equations is used. While for direct current EIT (that is ERT) the object is assumed surrounded by an insulator, in MIT one must account for the magnetic fields in the surrounding space, there being no magnetic 'shielding'.

We now come to the assumptions used to justify the usual mathematical model of EIT that are distinct from many other inverse problems for Maxwell's equations. We already have

Assumption 1 Transients components of all fields are negligible.

This assumption simply means we have waited a sufficient 'settling time' before making measurements.

We are interested in relatively low frequencies where magnetic effects can be neglected this translates in to two assumptions

Assumption $2 \omega \sqrt{\epsilon \mu}$ is small compared with the size of $\Omega$.

This means that the wavelength of propagating waves in the material is large. A measurement accuracy of $2^{-12}=1 / 4096$ is ambitious at higher frequencies means that for wave effects to be negligible

$$
d \omega \sqrt{\epsilon \mu}<\cos ^{-1} \frac{4095}{4096},
$$

where $d$ is the diameter of the body. Taking the relative permittivity to be 10 and $R=0.3 \mathrm{~m}$ gives a maximum frequency of $1 \mathrm{MHz}$.

Assumption $3 \sqrt{\omega \sigma \mu / 2}$ is small compared with the size of $\Omega$.

The quantity

$$
\delta=\sqrt{\frac{2}{\omega \sigma \mu}}
$$

is known as the skin depth. For a frequency of $10 \mathrm{kHz}$ and a conductivity of $0.5 \mathrm{Sm}^{-1}$ typical in medical applications, the skin depth is $7 \mathrm{~m}$. In geophysics lower frequencies are typical but length scales are larger. In a conducting cylinder the electric field decays with distance $r$ from the boundary at a rate $\mathrm{e}^{-r / \delta}$ due to the opposing magnetic field. At EIT frequencies this simple example 


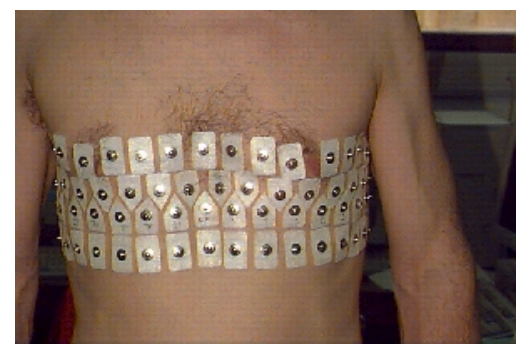

Figure 2: A system of eletrodes used for chest EIT at Oxford Brookes University. The positions of the electrodes was measured manually with a tape measure and the cross sectional shape was also determined by manual measurements. These electrodes have a disk of jell containing silver chloride solution that makes contact with the skin. Each eletrode was attached to the EIT system by a screened lead, not shown in this picture for clarity.

suggests that accurate forward modelling of EIT should take account of this effect although it is currently not thought to be a dominant source of error.

The effect of assumptions 2 and 3 combined together is that we can neglect $\nabla \times E$ in Maxwell's equations resulting in the standard equation for complex EIT

$$
\nabla \cdot(\sigma+\mathrm{i} \omega \epsilon) \nabla u=0
$$

Here the expression $\gamma=\sigma+i \omega \epsilon$ is called complex conductivity, or logically the admittivity, while $1 / \sigma$ is called resistivity and the rarely-used complex $1 / \gamma$ impedivity. A scaling argument is given for the approximation (0.1.11)in [33], and numerical checks on the validity of the approximation in [39] and [106].

It is often not so explicitly stated but, while in the direct current case one can neglect the conductivity of the air surrounding the body, for the alternating current case the electrodes are coupled capacitively and, while $\sigma$ can be assumed to be zero for air, the permittivity of any material is no smaller than that of a vacuum $\epsilon_{0}=8.85 \times 10^{-12}$, although dry air approaches that value. One requires then

Assumption $4 \omega \epsilon$ in the exterior is negligible compared to $|\sigma+i \omega \epsilon|$ in the interior.

For example, with a conductivity or $0.2 \mathrm{Sm}^{-1}$, the magnitude of the exterior admittivity reaches $2^{-12}$ of that value for a frequency of $0.88 \mathrm{MHz}$. For a more detailed calculation the capacitance between the electrodes externally could be compared with the impedance between electrodes. In ECT frequencies above $1 \mathrm{MHz}$ are used and the exterior capacitance can not be neglected. Indeed an exterior grounded shield is used so that the exterior capacitive coupling is not affected by the surroundings (see fig 3 ). 


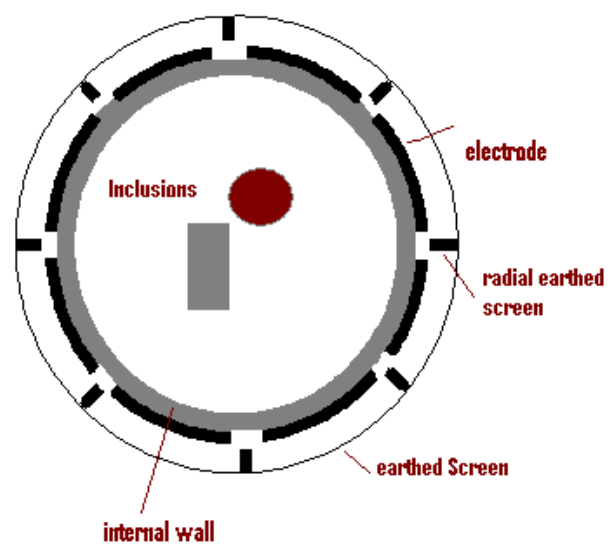

Figure 3: A cross section through a typical ECT sensor around a pipe (internal wall) showing the external screen with radial screens designed to reduce the external capacitive coupling between electrodes.

\subsubsection{The concentric anomaly: a simple example}

A simple example helps us to understand the instability in the inverse conductivity problem. Let $\Omega$ be the unit disk in $\mathbb{R}^{2}$ with polar coordinates $(r, \theta)$ and consider a concentric anomaly in the conductivity of radius $\rho<1$

$$
\gamma(x)= \begin{cases}a_{1}, & |x| \leq \rho \\ a_{0}, & \rho<|x| \leq 1\end{cases}
$$

From separation of variables, matching Dirichlet and Neumann boundary conditions at $|x|=\rho$, we find for $n \in \mathbb{Z}$

$$
\Lambda_{\gamma} \mathrm{e}^{\mathrm{i} n \theta}=|n| \frac{1+\mu \rho^{2|n|}}{1-\mu \rho^{2|n|}} \mathrm{e}^{\mathrm{i} n \theta},
$$

where $\mu=\left(a_{1}-a_{0}\right) /\left(a_{1}+a_{0}\right)$. From this one sees the effect of the Dirichlet to Neumann map on the complex Fourier series, and the effect on the real Fourier series is easily deduced. This example was considered in [65] as an example of the eigenvalues and eigenfunctions of $\Lambda_{\gamma}$, and also by [2] as an example of instability. We see that $\left\|\gamma-a_{0}\right\|_{L^{\infty}(\Omega)}=\left|a_{1}-a_{0}\right|$ independently of $\rho$ and yet $\Lambda_{\gamma} \rightarrow \Lambda_{a_{0}}$ in the operator norm. Hence if an inverse map $\Lambda_{\gamma} \mapsto \gamma$ exists, it cannot be continuous in this topology. Similar arguments can be used to show instability of inversion in other norms.

This example reveals many other features of the more general problem. For example experimentally one observes saturation: for an object placed away from the boundary, changes in the conductivity of an object with a conductivity close to the background are fairly easily detected, but for an object of very high or low conductivity further changes in conductivity of that object have little effect. This saturation effect was explored for offset circular objects (using conformal 
mappings) by Seagar[100]. This is also an illustration of the non-linearity of $\gamma \rightarrow \Lambda_{\gamma}$. One can also see in this example that smaller objects (with the same conductivity) produce smaller changes in measured data as one might expect.

On the unit circle $S^{1}$ one can define an equivalent norm on the Sobolev space $H_{\diamond}^{s}\left(S^{1}\right)$ (see definitions in Section 0.2.3) by

$$
\left\|\sum_{n=-\infty, n \neq 0}^{\infty} c_{n} m r e^{\mathrm{i} n \theta}\right\|_{s}^{2}=\sum_{n=-\infty, n \neq 0}^{\infty} n^{2 s} c_{n}^{2}
$$

It is clear for this example that $\Lambda_{\gamma}: H_{\diamond}^{s}\left(S^{1}\right) \rightarrow H^{s-1}\left(S^{1}\right)$, for any $s$. Roughly the current is a derivative of potential and one degree of differentiability less smooth. Technically $\Lambda_{\gamma}$ (for any positive $\gamma \in C^{\infty}(\Omega)$ ) is a first order pseudo-differential operator [82]. The observation that for our example $\mathrm{e}^{-\mathrm{i} n \theta} \Lambda_{\gamma} \mathrm{e}^{\mathrm{i} n \theta}=|n|+o\left(n^{-p}\right)$ as $|n| \rightarrow \infty$ for any $p>-1$ illustrates that the change in conductivity and radius of the interior object is of somewhat secondary importance! In the language of pseudodifferential operators for a general $\gamma$ such that $\gamma-1$ vanishes in a neighbourhood of the boundary, $\Lambda_{\gamma}$ and $\Lambda_{1}$ differ by a smoothing operator.

We see also from (0.1.13) that $\Lambda_{\gamma}^{-1}$ is also well defined operator on $L^{2} \rightarrow L^{2}$. with eigenvalues $O\left(|n|^{-1}\right)$ and is therefore a Hilbert-Schmidt operator. This is also known for the general case [38].

Early work on medical applications of EIT [60], [75] hoped that the forward problem in EIT would be approximated by generalized ray transform - that is integrals along current stream lines. The example of a concentric anomaly was used to illustrate that EIT is nonlocal [101]. If one applies the voltage $\cos (\theta+\alpha)$, which for a homogeneous disk would result in current streamlines that are straight and parallel, a change in conductivity in a small radius $\rho$ from the centre changes all measured currents, not just on lines passing through the region of changed conductivity $|x| \leq \rho$. In the 1980s a two dimensional algorithm that backprojected filtered data along equipotential lines was popularized by Barber and Brown [12]. Berenstein [15] later showed that the linearized EIT problem in a unit disc can be interpreted as the Radon transform with respect to the Poincaré metric and a convolution operator and that Barber and Brown's algorithm is an approximate inverse to this.

In process applications of EIT and related techniques the term soft field imaging is used, which by analogy to soft field X-rays means a problem that is non-linear and non-local. However in the literature when the 'soft field effect' is invoked, it is often not clear if it is the nonlinear or non local aspect to which they refer and in our opinion the term is best avoided.

\subsubsection{Measurements with electrodes}

A typical electrical imaging system uses a system of conducting electrodes attached to the surface of the body under investigation. One can apply current or voltage to these electrodes and measure voltage or current respectively. For one particular measurement the voltages (with respect to some arbitrary reference) are $V_{\ell}$ and the currents $I_{\ell}$, which we arrange in vectors as $\mathbf{V}$ and $\mathbf{I} \in \mathbb{C}^{L}$. 
The discrete equivalent of the Dirichlet-to-Neumann $\Lambda_{\gamma}$ map is the transfer admittance, or mutual admittance matrix $\mathbf{Y}$ which is defined by $\mathbf{I}=\mathbf{Y V}$.

It is easy to see that the vector $\mathbf{1}=(1,1, \ldots, 1)^{T}$ is in the null space of $\mathbf{Y}$, and that the range of $\mathbf{Y}$ is orthogonal to the same vector. Let $S$ be the subspace of $\mathbb{C}^{L}$ perpendicular to $\mathbf{1}$ then it can be shown that $\left.\mathbf{Y}\right|_{S}$ is invertible from $S$ to $S$. The generalized inverse $\mathbf{Z}=\mathbf{Y}^{\dagger}$ is called the transfer impedance. This follows from uniqueness of solution of shunt model boundary value problem.

The transfer admittance, or equivalently transfer impedance, represents a complete set of data which can be collected from the $L$ electrodes at a single frequency for a stationary linear medium. It can be seen from the weak formulation of (0.1.11) that $\mathbf{Y}$ and $\mathbf{Z}$ are symmetric (but for $\omega \neq 0$ not Hermittian). In electrical engineering this observation is called reciprocity. The dimension of the space of possible transfer admittance matrices is clearly no bigger than $L(L-1) / 2$, and so it is unrealistic to expect to recover more unknown parameters than this. In the analogous case of planar resistor networks with $L$ 'boundary' electrodes the possible transfer admittance matrices can be characterized completely [35], a characterization which is known at least partly to hold in the planar continuum case [64]. A typical electrical imaging system applies current or voltage patterns which form a basis of the space $S$, and measures some subset of the resulting voltages which as they are only defined up to an additive constant can be taken to be in $S$.

We have seen that the shunt model is non physical. In medical application with electrodes applied to skin and in "phantom" tanks used to test EIT systems with ionic solutions in contact with metal electrodes, a contact impedance layer exists between the solution or skin and the electrode. This modifies the shunting effect so that the voltage under the electrode is no longer constant. The voltage on the electrode is still a constant $V_{\ell}$ so now on $E_{\ell}$ there is a voltage drop across the contact impedance layer

$$
\phi+z_{\ell} \sigma \frac{\partial \phi}{\partial \nu}=V_{\ell},
$$

where the contact impedance $z_{\ell}$ could vary over $E_{\ell}$ but is usually assumed constant. Experimental studies have shown [58] that a contact impedance on each electrode is required for an accurate forward model. This new boundary condition together with (0.1.3) and (0.1.4) form the Complete Electrode Model or CEM. For experimental validation of this model see [32], theory [105] and numerical calculations $[97,114]$. A nonzero contact impedance removes the singularity in the current density, although high current densities still occur at the edges of the electrodes (fig 4). For further details on the singularity in the current density see [37]. While 'point electrodes', in which the current density is a sum of delta functions, are a limiting case of the CEM they are not physically realistic as they result in non-physical potentials not in $H^{1}(\Omega)$. The trace on the boundary cannot be evaluated at a point so point measurements of voltage are undefined. However it can be shown that the if the conductivity is changed only in the compliment of a neighbourhood of $\partial \Omega$ the resulting voltage difference at the boundary can be evaluated at points [52]. 

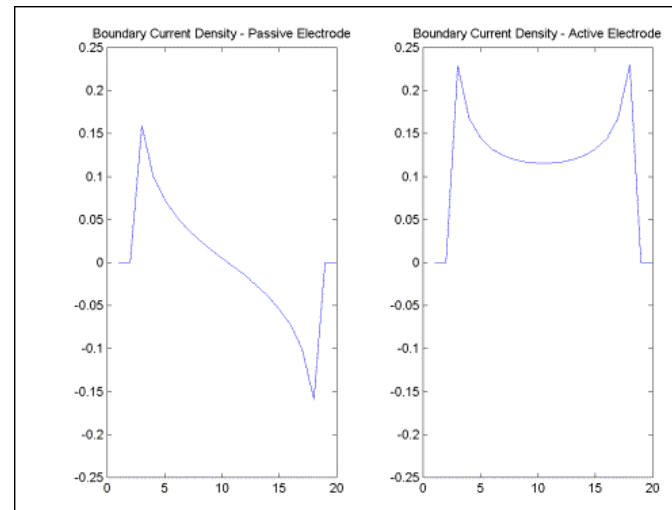

(a) Current density on the boundary for passive and active electrodes. In fact there is a jump discontinuity at the edge of electrodes for non-zeros contact impedance although our plotting routine has joined the left and right limits.

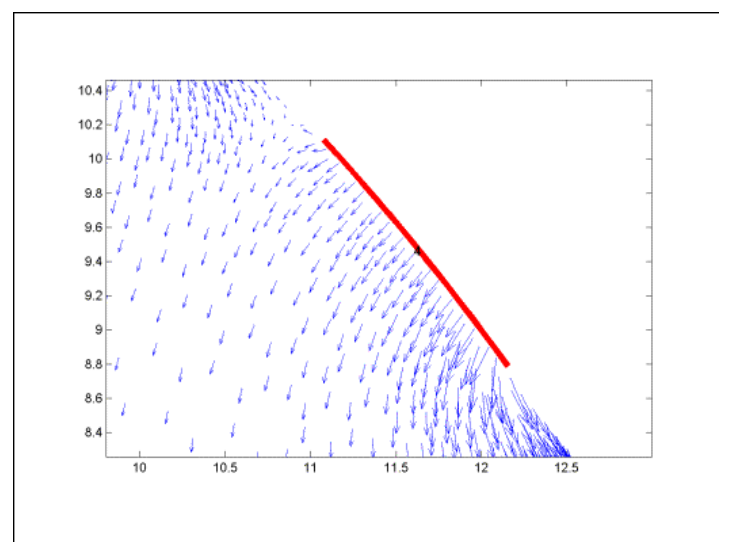

(c) Interior current flux near an active electrode.

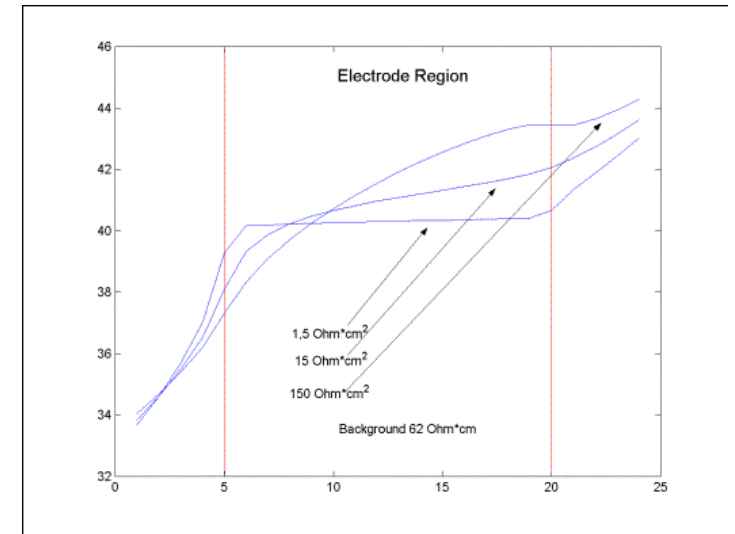

(b) The effect of contact impedance on the potential beneath an electrode. The potential is continuous.

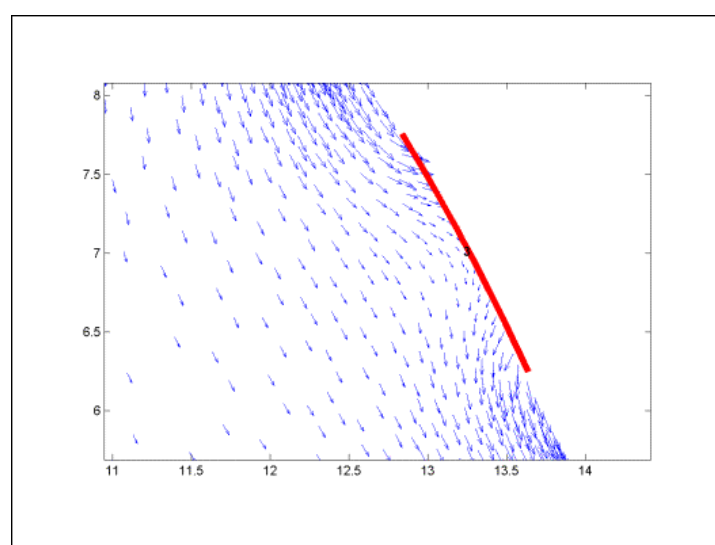

(d) Interior current flux near a passive electrode.

Figure 4: The current density on the boundary with the CEM is greatest at the edge of the electrodes, even for passive electrodes. This effect is reduced as the contact impedance increases. Diagrams courtesy of Andrea Borsic. 
The set of imposed current patterns, or excitation patterns, is designed to span $S$, or at least that part of it that can be accurately measured in a given situation. In medical EIT, with process ERT following suit, early systems designed at Sheffield [12] assumed a two dimensional circular domain. Identical electrodes were equally spaced on the circumference and, taking them to be numbered anticlockwise, the excitation patterns used were adjacent pairs, that is proportional to

$$
I_{\ell}^{i}=\left\{\begin{aligned}
1, & i=\ell \\
-1, & i=\ell+1 \\
0, & \text { otherwise },
\end{aligned}\right.
$$

for $i=1, \ldots L-1$. The electronics behind this is balanced current source connected between two electrodes [61, chapter 2]. and this is somewhat easier to achieve in practice than a variable current source at more than two electrodes. For general geometries, where the electrodes are not placed on a closed curve, other pairs of electrodes are chosen. For example $I_{1}^{i}=-1$, while $I_{\ell}^{i}=\delta_{i \ell}, \ell \neq 1$.

Measurements of voltage can only be differential and so voltage measurements are taken between pairs of electrodes, for example adjacent pairs, or between each and some fixed electrode. In pair drive systems, similar to the original Sheffield system, voltages on electrodes with nonzero currents are not measured, resulting in incomplete knowledge of $\mathbf{Z}$.

In geophysical surface resistivity surveys it is common to use a pair drive and pair measurement system, using electrodes in a line where a two dimensional approximation is used, or laid out in a rectangular or triangular grid where the full three dimensional problem is solved. Measurements taken between pairs of non-current carrying electrodes. The choice of measurement strategy is limited by the physical work involved in laying out the cables and by the switching systems. Often electrodes will be distributed along one line and a two dimensional approximate reconstruction used as this gives adequate information for less cost. A wider spacing of the current electrodes is used where the features of interest is located at a greater depth below the ground. In another geophysical configuration, cross borehole tomography, electrodes are deployed down several vertical cylindrical holes in the ground, typically filled with water, and current passed between electrodes in the same or between different bore holes. Surface electrodes may be used together with those in the bore holes. In some systems the current is measured to account for a non-ideal current source.

In capacitance tomography a basis of voltage patterns is applied and the choice $V_{\ell}^{i}=\delta_{i \ell}$ is almost universal. The projection of these vectors to $S$ (we call an 'electrode-wise basis') is convenient computationally as a current pattern.

Given a multiple drive system capable of driving an arbitrary vector of currents in $S$ (in practice with in some limits on the maximum absolute current and on the maximum voltage) we have a choice of excitation patterns. While exact measurements of $\mathbf{Z} \mathbf{I}^{i}$ for $\mathbf{I}^{i}$ in any basis for $S$ is clearly sufficient, the situation is more complicated with measurements of finite precision in the presence of noise. If a redundant set of currents is taken, the problem of estimating $\mathbf{Z}$ becomes one of multivariate linear regression. The choice of current patterns is then a 
design matrix. Another approach seeks the minimum set of current patterns that results in usable measurements. Applying each current pattern and taking a set of measurements takes a finite time, during which the admittivity changes. Without more sophisticated statistical methods (such as Kalman filters [115]), there are diminishing returns in applying redundant current patterns. Suppose that the total power $\mathbf{V}^{*} \mathbf{Z I}$ is constrained (we want to keep our patient electrically safe) and the current best estimate of the admittivity gives a transfer admittance $\mathbf{Z}_{\text {calc }}$, then it is reasonable to apply currents $\mathbf{I}$ such that $\left(\mathbf{Z}-\mathbf{Z}_{\text {calc }}\right) \mathbf{I}$ is above the threshold of voltages that can be accurately measured and modelled. One approach is to choose current patterns that are the right generalized singular vectors of $\mathbf{Z}-\mathbf{Z}_{\text {calc }}$ with singular values bigger than an error threshold. The generalized singular values are with respect to the norm $\left\|\mathbf{I}||_{\mathbf{Z}}:=\right\| \mathbf{Z I}||$ on $S$ and are the extrema of the distinguishability defined as

$$
\frac{\left\|\left(\mathbf{Z}-\mathbf{Z}_{\text {calc }}\right) \mathbf{I}\right\|}{\|\mathbf{I}\|_{\mathbf{Z}}},
$$

for $\mathbf{I} \in S$. These excitation patterns are called "optimal current patterns" [47] and can be calculated from an iterative procedure involving repeated measurement. For circular disk with rotationally symmetric admittivity and equally spaced identical electrodes, the singular vectors will be discrete samples of a Fourier basis and these trigonometric patterns are a common choice for multiple drive systems using a circular array of electrodes.

\subsection{Uniqueness of solution}

Uniqueness of solution is very important in inverse problems, although when talking to engineers it is often better to speak of sufficiency of data to avoid confusion. Interestingly it is generally true that results that show insufficiency of data, that one cannot recover an unknown function even if an infinite number of measurements of arbitrary precision are taken, have more impact in applied areas. While there are still unsolved problems in the uniqueness theory for the EIT inverse problem, there has been considerable progress over the last three decades and many important questions have been answered. While for an isotropic real conductivity $\gamma$ (with certain smoothness assumptions for dimensions $n \geq 3$ ), $\gamma$ is uniquely determined by the complete data $\Lambda_{\gamma}$ (see [11], [26], [109]), an anisotropic conductivity tensor is not uniquely determined by the boundary data, although some progress on what can be determined in this case has been made (see [3], [6], [45], [80]). Aside from knowing what can and cannot be determined with ideal data, there are two important ways the theoretical work has a practical impact. Firstly in some cases the proof of uniqueness of solution suggests a reconstruction algorithm. As we will see for the two-dimensional case the most effective approach (the so called $\bar{\partial}$-method) to uniqueness theory has now been implemented as a fast, practical algorithm. The other is an understanding of the instability and conditional stability of the 
inverse problem. This helps us to determine what a priori information is helpful in reducing the sensitivity of the solution to errors in the data.

In 1980 A. P. Calderón published a paper with the title 'On a inverse boundary value problem' [28], where he addressed the problem of whether it is possible to determine the conductivity of a body by making current and voltage measurements at the boundary. It seems that Calderón thought of this problem when he was working as an engineer in Argentina for the Yacimientos Petroliféros Fiscales (YPF), but it was only decades later that he decided to publish his results. This short paper is considered the first mathematical formulation of the problem. For a reprinted version of this manuscript we refer to [29]. The authors wish to recall also the work due to Druskin (see [40], [41], [42]) which has been carried on independently from Calderón's approach and has been devoted to the study of the problem from a geophysical point of view.

\subsubsection{The isotropic case}

\section{Calderón's paper}

Calderón considered a domain $\Omega$ in $\mathbb{R}^{n}, n \geq 2$, with Lipschitz boundary $\partial \Omega$. He took $\gamma$ be a real bounded measurable function in $\Omega$ with a positive lower bound. Let $Q_{\gamma}$ be the quadratic form (associated to $\Lambda_{\gamma}$ ) defined by

$$
Q_{\gamma}(\phi)=\left\langle\phi, \Lambda_{\gamma} \phi\right\rangle=\int_{\Omega} \gamma|\nabla u|^{2} d x
$$

where $u \in H^{1}(\Omega)$ solves the Dirichlet problem (0.1.2). Physically $Q_{\gamma}(\phi)$ is the Ohmic power dissipated when the boundary voltage $\phi$ is applied. The bilinear form associated with $Q_{\gamma}$ is then obtained by using the polarization identity

$$
\begin{aligned}
B_{\gamma}(\phi, \psi) & =\frac{1}{2}\left\{Q_{\gamma}(\phi+\psi)-Q_{\gamma}(\phi)-Q_{\gamma}(\psi)\right\} \\
& =\frac{1}{2}\left\{\int_{\Omega}\left(\gamma|\nabla(u+v)|^{2}-\gamma|\nabla u|^{2}-\gamma|\nabla v|^{2}\right) d x\right\} \\
& =\int_{\Omega} \gamma \nabla u \cdot \nabla v d x,
\end{aligned}
$$

where $L_{\gamma} v=0$ in $\Omega$ and $\left.v\right|_{\partial \Omega}=\psi \in H^{\frac{1}{2}}(\partial \Omega)$. Clearly a complete knowledge of any of $\Lambda_{\gamma}, Q_{\gamma}$ and $B_{\gamma}$ are equivalent. Calderón considered the 'forward' map

$$
\mathbf{Q}: \gamma \longrightarrow Q_{\gamma}
$$

and proved that $\mathbf{Q}$ is bounded and analytic in the subset of $L^{\infty}(\Omega)$ consisting of functions $\gamma$ which are real and have a positive lower bound. He then investigated the injectivity of the map and in order to do so, he linearized the problem. He in fact proved the injectivity of the Fréchet derivative of $\mathbf{Q}$ at $\gamma=1$. Here we will fill in a few details of the linearization for a general $\gamma$. Let $u$ be the solution 
to (0.1.2) and $U=u+w$ satisfy $L_{\gamma+\delta} U=0$, with $\left.U\right|_{\partial \Omega}=\phi$. The perturbation in potential satisfies $\left.w\right|_{\partial \Omega}=0$, we are considering the Dirichlet data fixed and investigating how the Neumann data varies when $\gamma$ is perturbed to $\gamma+\delta$. We have

$$
L_{\delta} u+L_{\gamma} w+L_{\delta} w=0 .
$$

Now let $G: H^{-1}(\Omega) \rightarrow H_{0}^{1}(\Omega)$ be the Green's operator that solves the equivalent of Poisson's equation for $L_{\gamma}$ with zero Dirichlet boundary conditions. That is for $g \in H^{-1}(\Omega), L_{\gamma} G g=g$ and $\left.G(g)\right|_{\partial \Omega}=0$ and we have the operator equation

$$
\left(1+G L_{\delta}\right) w=-G L_{\delta} u .
$$

An advantage of using the $L^{\infty}$ norm is that it is clear $\left\|L_{\delta}\right\| \rightarrow 0$ in the $H^{1}(\Omega) \rightarrow$ $H^{-1}(\Omega)$ operator norm as $\|\delta\|_{\infty} \rightarrow 0$. This means we can choose $\delta$ small enough that $\left\|G L_{\delta}\right\|<1$ (in the operator norm on $H^{1}(\Omega)$ ) and this ensures that the term in the bracket in (0.2.4) is invertible and the operator series in

$$
w=-\left(\sum_{k=1}^{\infty}\left(-G L_{\delta}\right)^{k}\right) u
$$

is convergent. This proves that the map $\gamma \mapsto u$ and hence $\mathbf{Q}$ is not just $C^{\infty}$ but analytic with (0.2.5) its Taylor series. We see immediately that the linearization of the map $\gamma \mapsto \Lambda_{\gamma}$ is

$$
\Lambda_{\gamma+\delta} \phi=\Lambda_{\gamma} \phi+\gamma \frac{\partial}{\partial \nu} G L_{\delta} u+\delta \frac{\partial u}{\partial \nu}+O\left(\|\delta\|_{\infty}^{2}\right) .
$$

A strength of this argument is that it gives the Fréchet derivative in these norms, rather than just the Gateaux derivative. It is easy to deduce that the Fréchet derivative of $\mathbf{Q}$ at $\gamma$ in the direction $\delta$ is given by

$$
d \mathbf{Q}(\gamma) \delta(\phi)=\int_{\Omega} \delta|\nabla u|^{2} d x
$$

In many practical situations it is more common to fix the Neumann boundary conditions and measure the change in boundary voltage as the conductivity changes. Suppose $L_{\gamma} u=0, L_{\gamma+\delta} U=0, w=U-u$ with

$$
\gamma \partial u / \partial \nu=(\gamma+\delta) \partial U / \partial \nu=g \in H^{-1 / 2}(\partial \Omega)
$$

then a similar argument to the above shows

$$
\int_{\partial \Omega} w \gamma \frac{\partial u}{\partial \nu} d x=-\int_{\Omega} \delta|\nabla u|^{2} d x+O\left(\|\delta\|_{\infty}^{2}\right) .
$$

The polarization identity is often applied to (0.2.8) giving

$$
\int_{\partial \Omega} w \gamma \frac{\partial v}{\partial \nu} d x=-\int_{\Omega} \delta \nabla u \cdot \nabla v d x+O\left(\|\delta\|_{\infty}^{2}\right),
$$


where $L_{\gamma} v=0$. This is often used in practice with

$$
\gamma \frac{\partial v}{\partial \nu}=\chi_{E_{i}} /\left|E_{i}\right|-\chi_{E_{j}} /\left|E_{j}\right|
$$

which represents the difference in the characteristic functions of a pair of electrodes. In the case of the shunt model this makes the left hand side of (0.2.8) the change in the difference between voltages on that pair of electrodes when the conductivity is perturbed. The formula (0.2.8) and its relatives are referred to as the Geselowitz Sensitivity Theorem in the bioengineering literature. With the complete electrode model (0.2.8) still holds, but with $u$ and $v$ satisfying $(0.1 .15)[99]$.

We now return to Calderón's argument: for $\gamma=1$ we have that $L_{1} u=\nabla^{2} u$. To prove the injectivity of $d \mathbf{Q}(1)$ we have to show that if the integral appearing in (0.2.7) vanishes for all the harmonic functions in $\Omega$, then $\delta=0$ in $\Omega$. Suppose the integral in (0.2.7) vanishes for all $u \in H^{1}(\Omega)$ such that $\nabla^{2} u=0$ in $\Omega$, then

$$
\int_{\Omega} \delta \nabla u \cdot \nabla v=0
$$

whenever $\nabla^{2} u=\nabla^{2} v=0$ in $\Omega$. For any $z \in \mathbb{R}^{n}$ consider $a \in \mathbb{R}^{n}$ such that $|a|=|z|, a \cdot z=0$ and consider the harmonic functions

$$
\begin{aligned}
& u(x)=e^{\pi i(z \cdot x)+\pi(a \cdot x)}, \\
& v(x)=e^{\pi i(z \cdot x)-\pi(a \cdot x)},
\end{aligned}
$$

which is equivalent to choosing

$$
u(x)=e^{x \cdot \rho}, \quad v(x)=e^{-x \cdot \bar{\rho}},
$$

where $\rho \in \mathbb{C}^{n}$ with

$$
\rho \cdot \rho=0 .
$$

Here we use the real dot product on complex vectors $\rho \cdot \rho:=\rho^{T} \rho$. With the choice made in $(0.2 .12),(0.2 .11)$ leads to

$$
2 \pi|z|^{2} \int \delta(x) e^{2 \pi i(z \cdot x)} d x=0, \quad \text { for each } \quad z,
$$

therefore $\delta(x)=0$, for all $x \in \Omega$. Calderón also observed that if the linear operator $d \mathbf{Q}(1)$ had a closed range, then one could have concluded that $Q$ itself was injective in a sufficiently small neighbourhood of $\gamma=$ constant. However conditions on the range of $d \mathbf{Q}(1)$, that would allow us to use the implicit function theorem, are either false or not known. Furthermore if the range was closed one could have also concluded that the inverse of $d \mathbf{Q}(1)$ was a bounded linear operator by the open mapping theorem. Calderón concluded the paper by giving an approximation for the conductivity $\gamma$ if 


$$
\gamma=1+\delta
$$

and $\delta$ is small enough in the $L^{\infty}$ norm, by making use of the same harmonic functions (0.2.12). Calderón's technique is based on the construction of low frequency oscillating solutions. Sylvester and Uhlmann proved in their fundamental paper [109] a result of uniqueness using high frequencies oscillating solutions of $L_{\gamma} u=0$. Their solutions are of type

$$
u(x, \xi, t)=e^{x \cdot \xi} \gamma^{-\frac{1}{2}}(1+\psi(x, \xi, t)),
$$

which behaves (for high frequencies $\xi$ ) in the same way as the solutions used by Calderón. These oscillating solutions have come to be known as complex geometrical optics (CGO) solutions. Before going in to more details of the use of CGO solutions we give an earlier result using a different approach.

\section{Uniqueness at the boundary}

In 1984 Kohn and Vogelius [76] proved that boundary values, and derivatives at the boundary, of a smooth isotropic conductivity $\gamma$ could be determined from the knowledge of $Q_{\gamma}$. Their result is given by the following theorem.

THEOREM 0.2.1. Let $\Omega$ be a domain in $\mathbb{R}^{n}(n \geq 2)$ with smooth boundary $\partial \Omega$. Suppose $\gamma_{i} \in C^{\infty}(\bar{\Omega}), i=1,2$ is strictly positive and that there is a neighborhood $B$ of some $x^{\star} \in \partial \Omega$ so that

$$
Q_{\gamma_{1}}(f)=Q_{\gamma_{2}}(f), \quad \text { for all } f, \quad f \in H^{\frac{1}{2}}(\partial \Omega), \quad \operatorname{supp}(f) \subset B .
$$

Then

$$
\frac{\partial^{|\alpha|}}{\partial x^{\alpha}} \gamma_{1}\left(x^{\star}\right)=\frac{\partial^{|\alpha|}}{\partial x^{\alpha}} \gamma_{2}\left(x^{\star}\right), \quad \forall \alpha .
$$

Theorem 0.2.1 is a local result in the sense that we only need to know $Q_{\gamma}$ in a open set of the boundary in order to determine the Taylor series of $\gamma$ on that open set. The global reformulation of this result given in terms of $\Lambda_{\gamma}$ is given below.

THEOREM 0.2.2. Let $\gamma_{i} \in C^{\infty}(\bar{\Omega}), i=1,2$ be strictly positive. If $\Lambda_{1}=\Lambda_{2}$, then

$$
\frac{\partial^{|\alpha|}}{\partial x^{\alpha}} \gamma_{1}=\frac{\partial^{|\alpha|}}{\partial x^{\alpha}} \gamma_{2}, \text { on } \partial \Omega, \quad \forall \alpha .
$$

For a sketch of the proof of theorem 0.2.2 see [112, Sketch of proof of theorem 4.1, pp 6].

This result settled the identifiability question in the real-analytic category of conductivities. Kohn and Vogelius have extended this result to piecewise realanalytic (piecewise constant, for example) conductivities in [77]. The proof of this result is based on [76] together with the Runge approximation theorem for solutions of $L_{\gamma} u=0$. 


\section{Complex geometrical optics solutions for the Schrödinger equation}

In 1987 Sylvester and Uhlmann [108], [109] constructed in dimension $n \geq 2$ complex geometrical optics solutions in the whole space for the Schrödinger equation with potential $q$. Before we state their result, the well known relation between the conductivity equation and the Schrödinger equation will be derived. This relationship is also important in diffuse optical tomography

LEMMA 0.2.3. Let $\gamma \in C^{2}(\bar{\Omega})$ be strictly positive then we have

$$
\gamma^{-\frac{1}{2}} L_{\gamma}\left(\gamma^{-\frac{1}{2}}\right)=\nabla^{2}-q
$$

where

$$
q=\frac{\nabla^{2}\left(\gamma^{\frac{1}{2}}\right)}{\gamma^{\frac{1}{2}}}
$$

Proof of Lemma 0.2.3.

$$
L_{\gamma} u=\gamma \nabla^{2} u+\nabla \gamma \cdot \nabla u
$$

therefore

$$
\gamma^{-\frac{1}{2}} L_{\gamma} u=\gamma^{\frac{1}{2}} \nabla^{2} u+\frac{\nabla \gamma \cdot \nabla u}{\gamma^{\frac{1}{2}}}
$$

Consider for $w=\gamma^{\frac{1}{2}} u$

$$
\begin{aligned}
\nabla^{2} w-q w & =\nabla^{2}\left(\gamma^{\frac{1}{2}} u\right)-\left(\nabla^{2} \gamma^{\frac{1}{2}}\right) u \\
& =\nabla \cdot\left(\nabla\left(\gamma^{\frac{1}{2}} u\right)\right)-\left(\nabla^{2} \gamma^{\frac{1}{2}}\right) u \\
& =\nabla \cdot\left(\left(\nabla \gamma^{\frac{1}{2}} u\right)+\gamma^{\frac{1}{2}}(\nabla u)\right)-\left(\nabla^{2} \gamma^{\frac{1}{2}}\right) u \\
& =\left(\nabla^{2} \gamma^{\frac{1}{2}}\right) u+2 \nabla \gamma^{\frac{1}{2}} \cdot \nabla u+\gamma^{\frac{1}{2}} \nabla^{2} u-\left(\nabla^{2} \gamma^{\frac{1}{2}}\right) u \\
& =\gamma^{\frac{1}{2}} \nabla^{2} u+\frac{\nabla \gamma \cdot \nabla u}{\gamma^{\frac{1}{2}}} \\
& =\gamma^{-\frac{1}{2}} L_{\gamma} u,
\end{aligned}
$$

which proves (0.2.13).

The term $q$ is usually called the potential of the Schrödinger equation, by analogy with the potential energy in quantum mechanics, this definition being somehow confusing given that in EIT $u$ is the electric potential. The results in [108], [109] state the existence of complex geometrical optics solutions for the Schrödinger equation with potential $q$ bounded and compactly supported in $\mathbb{R}^{n}$. We cite the result as given in [112], which relies on the weighted $L^{2}$ space $L_{\delta}^{2}\left(\mathbb{R}^{n}\right)=\{f$ : $\left.\int_{\mathbb{R}^{n}}\left(1+|x|^{2}\right)^{\delta}|f(x)|^{2} d x\right\}$. For $\delta<0$ this norm controls the "growth at infinity". The Sobolev spaces $H_{\delta}^{k}\left(\mathbb{R}^{n}\right)$ are formed in the standard way from $L_{\delta}^{2}\left(\mathbb{R}^{n}\right)$ 


$$
H_{\delta}^{k}\left(\mathbb{R}^{n}\right)=\left\{f \in W^{k}\left(\mathbb{R}^{n}\right) \mid D^{\alpha} f \in L_{\delta}^{2}\left(\mathbb{R}^{n}\right), \quad \text { for all }|\alpha| \leq k\right\},
$$

where $\alpha$ is a multi-index, $D^{\alpha} f$ denotes the $\alpha^{\text {th }}$ weak derivative of $f$ and $W^{k}\left(\mathbb{R}^{n}\right)$ is the set of $k$ times weakly differentiable functions on $\mathbb{R}^{n}$.

THEOREM 0.2.4. Let $q \in L^{\infty}\left(\mathbb{R}^{n}\right), n \geq 2$, with $q(x)=0$ for $|x| \geq R>0$ and $-1<\delta<0$. Then there exists $\epsilon(\delta)$ and such that for every $\rho \in \mathbb{C}^{n}$ satisfying

$$
\rho \cdot \rho=0
$$

and

$$
\frac{\left\|\left(1+|x|^{2}\right)^{1 / 2} q\right\|_{L^{\infty}\left(\mathbb{R}^{n}\right)}+1}{|\rho|} \leq \epsilon
$$

there exists a unique solution to

$$
\left(\nabla^{2}-q\right) u=0
$$

of the form

$$
u(x, \rho)=e^{x \cdot \rho}\left(1+\psi_{q}(x, \rho)\right),
$$

with $\psi_{q}(\cdot, \rho) \in L_{\delta}^{2}\left(\mathbb{R}^{n}\right)$. Moreover $\psi_{q}(\cdot, \rho) \in H_{\delta}^{2}\left(\mathbb{R}^{n}\right)$ and for $0 \leq s \leq 2$ there exists $C=C(n, s, \delta)>0$ such that

$$
\left\|\psi_{q}(\cdot, \rho)\right\|_{H^{2}} \leq \frac{C}{|\rho|^{1-s}} .
$$

Sketch of the proof of theorem 0.2.4. Let $u$ be a solution of (0.2.15) of type (0.2.16), then $\psi_{q}$ must satisfy

$$
\left(\nabla^{2}+2 \rho \cdot \nabla-q\right) \psi_{q}=q .
$$

The idea is that equation (0.2.18) can be solved for $\psi_{q}$ by constructing an inverse for $\left(\nabla^{2}+2 \rho \cdot \nabla\right)$ and solving the integral equation

$$
\psi_{q}=\left(\nabla^{2}+2 \rho \cdot \nabla\right)^{-1}\left(q\left(1+\psi_{q}\right)\right)
$$

for $\psi_{q}$. For more details about how to solve the above equation we refer to [112, Lemma 5.2] where it is shown that the integral equation (0.2.19) can only be solved in $L_{\delta}^{2}\left(\mathbb{R}^{n}\right)$ for large $|\rho|$.

Other approaches for the construction of complex geometrical optics solutions for the Schrödinger equation have been considered in [54], [68]. We refer to [112] for more details about references on this topic and a more in-depth explanation about the constructions of this kind of solutions. 


\section{Dirichlet-to-Neumann map and Cauchy data for the Schrödinger equa- tion}

If 0 is not a Dirichlet eigenvalue for the Schrödinger equation, then the Dirichletto-Neumann map associated to a potential $q$ can be defined by

$$
\tilde{\Lambda}_{q}(f)=\left.\frac{\partial u}{\partial \nu}\right|_{\partial \Omega}
$$

where $u$ solves the Dirichlet problem

$$
\left\{\begin{array}{l}
\left(\nabla^{2}-q\right) u=0 \quad \text { in } \Omega \\
\left.u\right|_{\partial \Omega}=f
\end{array}\right.
$$

As a consequence of lemma 0.2 .3 , for any $q=\frac{\nabla^{2} \gamma^{1 / 2}}{\gamma^{1 / 2}}$ we have

$$
\begin{aligned}
\tilde{\Lambda}_{q}(f) & =\left.\frac{\partial}{\partial \nu}\left(\gamma^{\frac{1}{2}} \gamma^{-\frac{1}{2}} u\right)\right|_{\partial \Omega} \\
& =\left.\left(\frac{\partial \gamma^{\frac{1}{2}}}{\partial \nu}\left(\gamma^{-\frac{1}{2}} u\right)+\gamma^{\frac{1}{2}} \frac{\partial\left(\gamma^{-\frac{1}{2}} u\right)}{\partial \nu}\right)\right|_{\partial \Omega} \\
& =\left.\left(\frac{1}{2} \gamma^{-\frac{1}{2}} \frac{\partial \gamma}{\partial \nu} \gamma^{-\frac{1}{2}}+\gamma^{\frac{1}{2}} \frac{\partial\left(\gamma^{-\frac{1}{2}} u\right)}{\partial \nu}\right)\right|_{\partial \Omega} \\
& =\left.\frac{1}{2}\left(\gamma^{-1} \frac{\partial \gamma}{\partial \nu}\right)\right|_{\partial \Omega} f+\left.\gamma^{\frac{1}{2}}\right|_{\partial \Omega} \Lambda_{\gamma}\left(\left.\gamma^{-\frac{1}{2}}\right|_{\partial \Omega} f\right) .
\end{aligned}
$$

So the two Dirichlet-to-Neumann maps $\tilde{\Lambda}_{q}$ and $\Lambda_{\gamma}$ are related in the following way

$$
\tilde{\Lambda}_{q}(f)=\left.\frac{1}{2}\left(\gamma^{-1} \frac{\partial \gamma}{\partial \nu}\right)\right|_{\partial \Omega} f+\left.\gamma^{\frac{1}{2}}\right|_{\partial \Omega} \Lambda_{\gamma}\left(\left.\gamma^{-\frac{1}{2}}\right|_{\partial \Omega} f\right),
$$

for any $f \in H^{\frac{1}{2}}(\partial \Omega)$. For $q \in L^{\infty}(\partial \Omega)$ we also define the Cauchy data as the set

$$
\mathbf{C}_{\mathbf{q}}=\left\{\left(\left.u\right|_{\partial \Omega},\left.\frac{\partial u}{\partial \nu}\right|_{\partial \Omega}\right) \mid u \in H^{1}(\Omega), \quad\left(\nabla^{2}-q\right) u=0 \quad \text { in } \Omega\right\} .
$$

If 0 is not an eigenvalue of $\nabla^{2}-q$, then $C_{q}$ is the graph given by

$$
\mathbf{C}_{\mathbf{q}}=\left\{\left(f, \tilde{\Lambda}_{q}(f)\right) \in H^{\frac{1}{2}}(\partial \Omega) \times H^{-\frac{1}{2}}(\partial \Omega)\right\} .
$$

What we saw so far is very general and holds in any dimension $n \geq 2$. We will distinguish in the rest of our discussion on the uniqueness of Calderón's problem between the higher dimensional case $n \geq 3$ and the two-dimensional one. 


\section{Global uniqueness for $n \geq 3$}

Sylvester and Uhlmann proved in [109] a result of global uniqueness for $C^{2}(\bar{\Omega})$ conductivities by solving in this way the identifiability question with the following result. Their result follows in dimension $n \geq 3$ from a more general one for the Schrödinger equation, which is useful in its own right for other inverse problems.

THEOREM 0.2.5. Let $q_{i} \in L^{\infty}(\Omega), i=1$, 2. Assume $C_{q_{1}}=C_{q_{2}}$, then $q_{1}=q_{2}$.

Proof of theorem 0.2.5. This result has been proved by constructing oscillatory solutions of $\left(\nabla^{2}-q_{i}\right) u_{i}=0$ in $\mathbb{R}^{n}$ with high frequencies. We start by stating that the following equality

$$
\int_{\Omega}\left(q_{1}-q_{2}\right) u_{1} u_{2}=0
$$

is true for any $u_{i} \in H^{1}(\Omega)$ solution to

$$
\left(\nabla^{2}-q_{i}\right) u_{i}=0 \quad \text { in } \quad \Omega, \quad i=1,2 .
$$

Equality (0.2.21) follows by

$$
\int_{\Omega}\left(q_{1}-q_{2}\right) u_{1} u_{2}=\int_{\partial \Omega}\left(\frac{\partial u_{1}}{\partial \nu} u_{2}-u_{1} \frac{\partial u_{2}}{\partial \nu}\right) d S
$$

which can be easily obtained by the divergence theorem. We extend $q_{i}$ on the whole $\mathbb{R}^{n}$ by taking $q_{i}=0$ on $\mathbb{R}^{n} \backslash \Omega$ and we take solutions of

$$
\left(\nabla^{2}-q_{i}\right) u_{i}=0 \quad \text { in } \quad \mathbb{R}^{n}, \quad i=1,2
$$

of the form

$$
u_{i}=e^{x \cdot \rho_{i}}\left(1+\psi_{q_{i}}\left(x, \rho_{i}\right)\right), \quad i=1,2,
$$

with $\left|\rho_{i}\right|$ large. This type of solutions are known as complex geometrical optics solutions. $\rho_{i}, i=1,2$ is chosen of type

$$
\begin{aligned}
& \rho_{1}=\frac{\eta}{2}+i\left(\frac{k+l}{2}\right) \\
& \rho_{2}=-\frac{\eta}{2}+i\left(\frac{k-l}{2}\right),
\end{aligned}
$$

with $\eta, k, l \in \mathbb{R}^{n}$ and satisfying

$$
\eta \cdot k=k \cdot k=\eta \cdot l=0, \quad|\eta|^{2}=|k|^{2}+|l|^{2},
$$


the choices of $\eta, k, l$ having been made so that $\rho_{i} \cdot \rho_{i}=0, i=1,2$. With these choices of $\rho_{i}, i=1,2$ we have

$$
\begin{aligned}
u_{1} u_{2} & =\left[e^{x \cdot \frac{\eta}{2}+i x \cdot\left(\frac{k+l}{2}\right)}+e^{x \cdot \frac{\eta}{2}+i x \cdot\left(\frac{k+l}{2}\right)} \psi_{q_{1}}\right] \cdot\left[e^{-x \cdot \frac{\eta}{2}+i x \cdot\left(\frac{k-l}{2}\right)}+e^{-x \cdot \frac{\eta}{2}+i x \cdot\left(\frac{k-l}{2}\right)} \psi_{q_{2}}\right] \\
& =e^{i x \cdot k}\left(1+\psi_{q_{1}}+\psi_{q_{2}}+\psi_{q_{1}} \psi_{q_{2}}\right)
\end{aligned}
$$

and therefore

$$
\left(\widehat{q_{1}-q_{2}}\right)(-k)=-\int_{\Omega} e^{i x \cdot k}\left(q_{1}-q_{2}\right)\left(\psi_{q_{1}}+\psi_{q_{2}}+\psi_{q_{1}} \psi_{q_{2}}\right) d x
$$

By recalling that

$$
\left\|\psi_{q_{i}}\right\|_{L^{2}(\Omega)} \leq \frac{C}{\left|\rho_{i}\right|}
$$

and letting $|l| \rightarrow \infty$ we obtain $q_{1}=q_{2}$ (see [112, proof of theorem 6.2 , pp $10])$.

As a consequence of this result we finally obtain result [109] stated here below.

THEOREM 0.2.6. Let $\gamma_{i} \in C^{2}(\bar{\Omega})$, $\gamma_{i}$ strictly positive, $i=1$, 2. If $\Lambda_{\gamma_{1}}=\Lambda_{\gamma_{2}}$, then $\gamma_{1}=\gamma_{2}$ in $\bar{\Omega}$.

Theorem 0.2.6 has been proved in [109] in a straightforward manner by constructing highly oscillatory solutions to $L_{\gamma} u=0$ in $\Omega$. In this chapter we follow the line of [112] in the exposition of such result as a consequence of the more general theorem 0.2.5. Such a choice has been made because of the clearer exposition made in [112].

We will proceed by showing that theorem 0.2.5 implies theorem 0.2.6 for sake of completeness. The reader can find it also in [112]. The argument used is the following. Let $\gamma_{i} \in C^{2}(\bar{\Omega})$ be strictly positive and $\Lambda_{\gamma_{1}}=\Lambda_{\gamma_{2}}$. Then by [76] we have

$$
\begin{aligned}
\left.\gamma_{1}\right|_{\partial \Omega} & =\left.\gamma_{2}\right|_{\partial \Omega}, \\
\left.\frac{\partial \gamma_{1}}{\partial \nu}\right|_{\partial \Omega} & =\left.\frac{\partial \gamma_{2}}{\partial \nu}\right|_{\partial \Omega},
\end{aligned}
$$

therefore (0.2.20) implies $C_{q_{1}}=C_{q_{2}}$ i.e. $q_{1}=q_{2}=: q$ because of theorem 0.2.5. Recall that

$$
q_{i}=\frac{\nabla^{2} \gamma_{i}^{1 / 2}}{\gamma_{i}^{1 / 2}}, \quad i=1,2
$$

which leads to

$$
\begin{aligned}
& \nabla^{2} \gamma_{1}^{\frac{1}{2}}-q \gamma_{1}^{\frac{1}{2}}=0 \\
& \nabla^{2} \gamma_{2}^{\frac{1}{2}}-q \gamma_{2}^{\frac{1}{2}}=0
\end{aligned}
$$


i.e.

$$
\nabla^{2}\left(\gamma_{1}^{\frac{1}{2}}-\gamma_{2}^{\frac{1}{2}}\right)-q\left(\gamma_{1}^{\frac{1}{2}}-\gamma_{2}^{\frac{1}{2}}\right)=0
$$

with

$$
\left.\left(\gamma_{1}^{\frac{1}{2}}-\gamma_{2}^{\frac{1}{2}}\right)\right|_{\partial \Omega}=0
$$

Therefore it must be that

$$
\gamma_{1}=\gamma_{2} \quad \text { in } \Omega,
$$

by uniqueness of the solution of the Cauchy problem.

The identifiability question was then pushed forward to he case of $\gamma \in$ $C^{1,1}(\bar{\Omega})$ with an affirmative answer by Nachman, Sylvester and Uhlmann in 1988 [92]. Nachman extended then this result to domains with $C^{1,1}$ boundaries (see [90]). The condition on the boundary was relaxed to $\partial \Omega$ Lipschitz by Alessandrini in 1990 in [3]; he proved uniqueness at the boundary and gave stability estimates for $\gamma \in W^{1, p}(\Omega)$, with $p>n$ by making use of singular solutions with an isolated singularity at the centre of a ball. This method enables one to construct solutions of $L_{\gamma} u=0$ on a ball behaving asymptotically like the singular solutions of the Laplace-Beltrami equation with separated variables. His results hold in dimension $n \geq 2$. Results of global uniqueness in the interior were also found in [3] among piecewise analytic perturbations of $\gamma$, giving an extension of Kohn and Vogelius result in [77] to Lipschitz domains.

Going back to the issue of global uniqueness, Brown [25] relaxed the regularity of the conductivity to $\frac{3}{2}+\varepsilon$ derivatives, which was followed by the uniqueness result of Päivärinta-Pachenko-Uhlmann [98] for $W^{\frac{3}{2}, \infty}$ conductivities. Their result is based on the construction of complex geometrical optics (CGO) solutions for conductivities $\gamma \in W^{1, \infty}\left(\mathbb{R}^{n}\right)(n \geq 2)$. We recall in what follow their construction of the CGO followed by their uniqueness result.

THEOREM 0.2.7. [98] Let $\gamma \in W^{1, \infty}\left(\mathbb{R}^{\ltimes}\right), \gamma$ strictly positive and $\gamma=1$ outside a large ball. Let $-1<\delta<0$, then for $|\rho|$ sufficiently large there is a unique solution of

$$
\operatorname{div}(\gamma \nabla u)=0 \quad \text { in } \quad \mathbb{R}^{\ltimes}
$$

of the form

$$
u=e^{x \cdot \rho}\left(\gamma^{-\frac{1}{2}}+\psi_{\gamma}(x, \rho)\right)
$$

with $\psi_{\gamma} \in L_{\delta}^{2}\left(\mathbb{R}^{n}\right)$. Moreover, $\psi_{\gamma}$ has the form

$$
\psi_{\gamma}(x, \rho)=\left(\omega_{0}(x, \rho)-\gamma^{-\frac{1}{2}}\right)+\omega_{1}(x, \rho),
$$

where $\omega_{0}, \omega_{1}$ satisfy

$$
\lim _{|\rho| \rightarrow \infty}\left\|\omega_{0}(x, \rho)-\gamma^{-\frac{1}{2}}\right\|_{H_{\delta}^{1}}=0
$$


and

$$
\lim _{|\rho| \rightarrow \infty}\left\|\omega_{1}(x, \rho)\right\|_{L_{\delta}^{2}}=0
$$

Here we want to recall the idea behind the proof of the above Theorem 0.2.7. The first step is to rewrite the conductivity equation

$$
\operatorname{div}(\gamma \nabla u)=0 \quad \text { in } \quad \mathbb{R}^{n}
$$

as

$$
\Delta u+A \cdot \nabla u=0 \quad \text { in } \quad \mathbb{R}^{n},
$$

where

$$
A+\nabla \log \gamma \in L^{\infty}\left(\mathbb{R}^{n}\right)
$$

has compact support. By introducing

$$
\Phi_{\varepsilon}=\varepsilon^{-n}\left(\frac{x}{\varepsilon}\right),
$$

with $\Phi(x)$ a mollifier, one can define

$$
\begin{aligned}
\varphi_{\varepsilon} & =\Phi_{\varepsilon} * \log \gamma \\
A_{\varepsilon} & =\Phi_{\varepsilon} * A \\
\omega_{0}(x, \varepsilon) & =e^{-\frac{\varphi_{\varepsilon}(x)}{2}}
\end{aligned}
$$

and with the above choice of $\omega_{0}$ one can show that

$$
\lim _{\varepsilon \rightarrow 0}\left\|\omega_{0}-\gamma^{-\frac{1}{2}}\right\|_{H_{\delta}^{1}}=0 .
$$

Let $\rho \in \mathbb{C}^{n}$ be such that $\rho \cdot \rho=0$ and define the operators

$$
\begin{aligned}
\Delta_{\rho} u:=e^{-x \cdot \rho} \Delta\left(e^{x \cdot \rho} u\right) & =\Delta u+2 \rho \cdot \nabla u \\
\nabla_{\rho} u:=e^{-x \cdot \rho} \nabla\left(e^{x \cdot \rho} u\right) & =\nabla u+\rho u .
\end{aligned}
$$

One can then define for any $f \in C_{0}^{\infty}\left(\mathbb{R}^{n}\right)$

$$
\Delta_{\rho}^{-1} f=\frac{1}{(2 \pi)^{n}} \int e^{i x \cdot \xi \frac{\hat{f}(\xi)}{-|\xi|^{2}+2 i \rho \cdot \xi}} d \xi,
$$

which can then be extended to a bounded operator

$$
\Delta_{\rho}^{-1}: H_{\delta+1}^{s}\left(\mathbb{R}^{n}\right) \rightarrow H_{\delta}^{s}\left(\mathbb{R}^{n}\right)
$$

for any $-1<\delta<0$ and $s \geq 0$. Moreover 


$$
\left\|\Delta_{\rho}^{-1}\right\|_{H_{\delta+1}^{s} \rightarrow H_{\delta}^{s}} \leq \frac{C(s, \delta, n)}{|\rho|}
$$

for some $C>0$. The idea is now to construct $\omega_{1}$ solution to

$$
\left(\Delta_{\rho}+A \cdot \nabla_{\rho}\right) \omega_{1}=-\left(\Delta_{\rho}+A \cdot \nabla_{\rho}\right) \omega_{0},
$$

where we recall that $\omega_{0}(x, \varepsilon)=e^{-\frac{\varphi_{\varepsilon}(x)}{2}}$ and therefore depends on $\varepsilon$. If we set now

$$
\omega_{1}=\Delta_{\rho}^{-1} \tilde{\omega}
$$

we can then rewrite $(0.2 .33)$ as

$$
\underbrace{\left(I+A \cdot \nabla_{\rho} \Delta_{\rho}^{-1}\right)}_{:=T_{\rho}(\gamma)} \tilde{\omega}=f_{\varepsilon}
$$

where

$$
\begin{aligned}
f_{\varepsilon} & :=-\left(\Delta_{\rho}+A \cdot \nabla_{\rho}\right) \omega_{0} \\
& =-e^{-\frac{\varphi_{\varepsilon}}{2}}\left(-\frac{1}{2} \Delta \varphi_{\varepsilon}+\frac{1}{4}\left(\nabla \varphi_{\varepsilon}\right)^{2}-\frac{1}{2} A \cdot \nabla \varphi_{\varepsilon}+\left(A-A_{\varepsilon}\right) \cdot \rho\right) .
\end{aligned}
$$

An approximate inverse of $T_{\rho}(\gamma)$ is given by

$$
\begin{aligned}
S_{\rho}(\gamma) & :=\gamma^{-\frac{1}{2}}\left(I-A \cdot \nabla_{\rho} \delta_{\rho}^{-1}\right) \gamma^{\frac{1}{2}} \\
& =\gamma^{-\frac{1}{2}} T_{\rho}\left(\gamma^{-1}\right) \gamma^{\frac{1}{2}},
\end{aligned}
$$

therefore (0.2.34) has a unique solution in an appropriate space. To study now the behavior of $\omega_{1}$ as $\varepsilon \rightarrow 0$ and $|\rho| \rightarrow \infty$, we recall that

$$
\omega_{1}(x, \varepsilon, \rho)=\Delta_{\rho}^{-1} S_{\rho} f_{\varepsilon}+\underbrace{\Delta_{\rho}^{-1}\left(T_{\rho}^{-1}-S_{\rho}\right) f_{\varepsilon}}_{:=h_{\rho}}
$$

and now one can show that

$$
\lim _{|\rho| \rightarrow \infty}\left\|h_{\rho}\right\|_{H_{\delta}^{1}\left(\mathbb{R}^{n}\right)}=0,
$$

which concludes the proof. Theorem 0.2.26 is then used in [98] to prove global uniqueness for conductivities $\gamma \in W^{\frac{3}{2}, \infty}(\Omega)$, where $\Omega$ is a bounded domain in $\mathbb{R}^{n}$, with $n \geq 3$. More precisely they prove the following theorem. 
THEOREM 0.2.8. Let $n \geq 3$. Let $\gamma_{i} \in W^{\frac{3}{2}, \infty}(\Omega)$ be strictly positive on $\bar{\Omega}$, $i=1,2$. If

$$
\Lambda_{\gamma_{1}}=\Lambda_{\gamma_{2}}
$$

then

$$
\gamma_{1}=\gamma_{2} \quad \text { on } \quad \Omega
$$

Again we just want to give to the reader a flavor of how Theorem 0.2.8 is proven in [98]. The main idea is that if $\gamma_{i} \in W^{1, \infty}(\Omega)$ and $\Lambda_{\gamma_{1}}=\Lambda_{\gamma_{2}}$, with $a_{i}=\sqrt{\gamma_{i}}, i=1,2$, then the identity

$$
\int_{\Omega}\left(\nabla a_{1} \cdot \nabla\left(u_{1} v\right)-\nabla a_{2} \cdot \nabla\left(u_{2} v\right)\right)-\int_{\Omega}\left(\nabla\left(a_{1} u_{1}\right) \cdot \nabla v-\nabla\left(a_{2} u_{2}\right) \cdot \nabla v\right) d x=0,
$$

holds true $\forall v \in H^{1}(\Omega), \forall u_{i} \in H^{1}(\Omega)$ solution to $\operatorname{div}\left(\gamma_{i} \nabla u_{i}\right)=0$ in $\Omega$, $=1,2$. The reader should notice that so far the results obtained hold true for conductivities of type $\gamma \in W^{1, \infty}(\Omega)$. It is at this stage that one needs to assume

$$
\gamma \in W^{\frac{3}{2}, \infty}\left(\mathbb{R}^{n}\right)
$$

to show the following technical results for $\omega_{1}(x, \rho)=\omega_{1}(x, \varepsilon, \rho)$ as in (0.2.35), $\varepsilon=|\rho|^{-1}$

$$
\lim _{|\rho| \rightarrow \infty} \int e^{i x \cdot \xi} \nabla \gamma^{\frac{1}{2}} \cdot \nabla \omega_{1} d x=0
$$

With this choice of $\omega_{1}$, by substituting the complex geometrical optics solutions (0.2.27) into identity (0.2.37) one then can gain the desired uniqueness result.

The above result was then followed by uniqueness for $W^{\frac{3}{2}, p}$ (with $p>2 n$ ) in [24]. Recently Haberman and Tataru [50] proved that uniqueness holds for $C^{1}$ conductivities and Lipschitz conductivities close to the identity. Their result is the following.

THEOREM 0.2.9. Let $n \geq 3$ and $\Omega \subset \mathbb{R}^{n}$ be a bounded domain with Lipschitz boundary. Let $\gamma_{i} \in W^{1, \infty}(\bar{\Omega})$ be a real conductivity, $i=1,2$. Suppose there exists a constant $C=C(n, \Omega)$ such that $\gamma_{i}, i=1,2$ satisfies either

$$
\left\|\nabla \log \gamma_{i}\right\|_{L^{\infty}(\bar{\Omega})} \leq C
$$

or

$$
\gamma_{i} \in C^{1}(\bar{\Omega}) .
$$

If

$$
\Lambda_{\gamma_{1}}=\Lambda_{\gamma_{2}}
$$


then

$$
\gamma_{1}=\gamma_{2}
$$

\section{Global uniqueness in the two-dimensional case}

The two-dimensional inverse conductivity problem must often be treated as a special case. Although results in [77] gave a positive answer to the identifiability question in the case of piecewise analytic conductivities, it was not until 1996 that Nachman [91] proved a global uniqueness result to Calderón problem for conductivities in $W^{2, p}(\Omega)$, for some $p>1$. An essential part of his argument is based on the construction of the complex geometrical optics solutions and the $\bar{\partial}$-method (sometimes written 'd-bar method') in inverse scattering introduced in one dimension by Beals and Coifman (see [22], [23]). The result of [91] has been improved in 1997 for conductivities having one derivative in an appropriate sense (see [26]) and the question of uniqueness was settled in $L^{\infty}(\Omega)$ finally by Astala and Päivärinta [11] using $\bar{\partial}$-methods. They proved

THEOREM 0.2.10. Let $\Omega$ be a bounded domain in $\mathbb{R}^{2}$ and $\gamma_{i} \in L^{\infty}, i=1,2$ be real functions such that for some constant $M, M^{-1}<\gamma_{i}<M$. Then

$$
\Lambda_{\gamma_{1}}=\Lambda_{\gamma_{2}} \Longrightarrow \gamma_{1}=\gamma_{2} .
$$

Let us first explain the complex version of the problem used by [11]. We use the complex variable $z=x_{1}+\mathrm{i} x_{2}$, use the notation $\partial=\partial / \partial z, \bar{\partial}=\partial / \partial \bar{z}$. Then we have the following result [11]:

LEMMA 0.2.11. Let $\Omega$ be the unit disk in the plane and $u \in H^{1}(\Omega)$ be a solution of $L_{\gamma} u=0$. Then there exists a real function $v \in H^{1}(\Omega)$, unique up to a constant, such that $f=u+\mathrm{i} v$ satisfies the Beltrami equation

$$
\bar{\partial} f=\mu \overline{\partial f},
$$

where $\mu=(1-\gamma) /(1+\gamma)$.

Conversely if $f \in H^{1}(\Omega)$ satisfies (0.2.41), with a real valued $\mu$, then $u=$ $\operatorname{Re} f$ and $v=\operatorname{Im} f$ satisfy

$$
L_{\gamma} u=0 \quad \text { and } \quad L_{\gamma^{-1}} v=0,
$$

where $\gamma=(1-\mu) /(1+\mu)$.

Astala and Päivärinta reduce the general case of $\Omega$ to that of the disk, and show that the generalized Hilbert transform $\mathcal{H}_{\mu}:\left.\left.u\right|_{\partial \Omega} \mapsto v\right|_{\partial \Omega}$ uniquely determines, and is determined by $\Lambda_{\gamma}$. They go on to construct CGO solutions to $(0.2 .41)$ of the form

$$
f_{\mu}(z, k)=\mathrm{e}^{\mathrm{i} k z}\left(1+O\left(\frac{1}{z}\right)\right) \text { as }|z| \rightarrow \infty
$$


and using a result connecting pseudoanalytic functions with quasi-regular maps prove that $\mathcal{H}_{\mu}$ determines $\mu$. The original method Nachman used to prove uniqueness has resulted in the development of $\bar{\partial}$ reconstruction methods which are described below (section 0.3.5). See also the work of Druskin [40], which provides some answers to the 2-D geophysical settings.

\section{Some open problems for the uniqueness}

One of the main open problems in dimension $n \geq 3$ is to investigate whether global uniqueness holds for the minimal assumption $\gamma \in L^{\infty}(\Omega)$ or else to find what are the minimal assumptions on $\gamma$ in order to guarantee uniqueness. The inverse conductivity problem makes sense for conductivities that are indeed merely $L^{\infty}$. There are neither proofs nor counter-examples for this in any dimension as far as we know but it has been conjectured by Uhlmann that the optimal assumption is that the conductivities are Lipschitz. These open problems influence of course also the stability issue of finding appropriate assumptions (possibly on $\gamma$ ) in order to improve the unstable nature of EIT. This issue will be study in the next section.

\section{Stability of the solution at the boundary}

The result of uniqueness at the boundary of theorem 0.2 .2 has been improved in [110] to a stability estimate. The result is the following.

THEOREM 0.2.12. Let $\gamma_{i} \in C^{\infty}(\bar{\Omega}), i=1,2$, satisfy

$$
\begin{aligned}
& 0<\frac{1}{E} \leq \gamma_{i} \leq E, \quad i=1,2 \\
& \left\|\gamma_{i}\right\|_{C^{2}(\bar{\Omega})} \leq E, \quad i=1,2 .
\end{aligned}
$$

Given any $0<\sigma<\frac{1}{n+1}$, there exists $C=C(\Omega, E, n, \sigma)$ such that

$$
\left\|\gamma_{1}-\gamma_{2}\right\|_{L^{\infty}(\partial \Omega)} \leq C|| \Lambda_{\gamma_{1}}-\Lambda_{\gamma_{2}} \|_{*}
$$

and

$$
\left\|\frac{\partial \gamma_{1}}{\partial \nu}-\frac{\partial \gamma_{2}}{\partial \nu}\right\|_{L^{\infty}(\partial \Omega)} \leq C\left\|\Lambda_{\gamma_{1}}-\Lambda_{\gamma_{2}}\right\|_{*}^{\sigma}
$$

where $\|\cdot\|_{*}$ denotes the norm in the space of bounded linear operators from $H^{\frac{1}{2}}(\partial \Omega)$ to $H^{-\frac{1}{2}}(\partial \Omega)$.

This result improves the one of theorem 0.2.2 in the sense that we no longer require that $\gamma \in C^{\infty}(\bar{\Omega})$ to determine $\gamma$ itself and its derivative at the boundary. We only need $\gamma$ to be continuous on $\bar{\Omega}$ to determine the boundary values of $\gamma$, where if $\gamma \in C^{1}(\bar{\Omega})$ then we can determine $\gamma$ and its first derivative on $\partial \Omega$ as well. Subsequent results of stability at the boundary along the same lines have been proved in [3], [7], [25], [45], [90] and [93]. 
Global stability for $n \geq 3$

In 1988 Alessandrini [2] proved that, in dimension $n \geq 3$, under an a priori assumption on $\gamma$ of type

$$
\|\gamma\|_{H^{s}(\Omega)} \leq E, \quad \text { for some } s>\frac{n}{2}+2,
$$

$\gamma$ depends continuously on $\Lambda_{\gamma}$ with a modulus of continuity of logarithmic type. The result is stated below.

THEOREM 0.2.13. Let $n \geq 3$. Suppose that $s>\frac{n}{2}$ and that $\gamma_{i} \in C^{\infty}(\bar{\Omega})$, $i=1,2$ is a conductivity satisfying

$$
\begin{array}{ll}
0<\frac{1}{E} \leq \gamma_{i} \leq E, & i=1,2 \\
\left\|\gamma_{i}\right\|_{H^{s+2}(\Omega)} \leq E, & i=1,2 .
\end{array}
$$

Then there exists $C=C(\Omega, E, n, s)$ and $\tau=\tau(n, s)$, with $0<\tau<1$ such that

$$
\left\|\gamma_{1}-\gamma_{2}\right\|_{L^{\infty}(\Omega)} \leq C\left(\left.\left.|\log |\left|\Lambda_{\gamma_{1}}-\Lambda_{\gamma_{2}}\right|\right|_{*}\right|^{-\tau}+|| \Lambda_{\gamma_{1}}-\Lambda_{\gamma_{2}} \|_{*}\right) .
$$

It has been proved [3], [4] that a similar stability estimate holds if (0.2.49) is replaced by

$$
\left\|\gamma_{i}\right\|_{W^{2, \infty}(\Omega)} \leq E, \quad i=1,2 .
$$

Mandache [87] proved that logarithmic stability is optimal for dimension $n \geq 2$ if the a priori assumption is of the form

$$
\|\gamma\|_{C^{k}(\bar{\Omega})} \leq E
$$

for any finite $k=0,1,2, \ldots$. One of the main open problems in the stability issue is then to improve this logarithmic-type stability estimate under some additional a priori condition. In [8] it has been shown that (0.2.50) can be improved to a Lipschitz-type estimate in the case in which $\gamma$ is piecewise constant with jumps on a finite number of domains. For piecewise constant complex conductivities a similar result has been proved in [19], where piecewise constant potentials of the Schrödinger equation have been investigated in [20] and Lipschitz stability estimates have been proved in this case as well. We refer to [5] for a more in depth discussion about the stability in EIT and open problems in that regard. A similar estimate to (0.2.50) for the potential case can be found in [112].

\section{Global stability for the two-dimensional case}

Logarithmic-type stability estimates in dimension $n=2$ were obtained by [13] and [14], [83]. The results obtained in the last require only $\gamma$ be Hölder continuous of positive exponent 


$$
\|\gamma\|_{C^{\alpha}(\bar{\Omega})} \leq E
$$

for some $\alpha, 0<\alpha \leq 1$.

\section{Some open problems for the stability}

The main open problem is to improve the logarithmic-type estimate found in [2] in any dimension $n \geq 2$. One approach would be to investigate whether the a priori regularity assumptions (0.2.51) can be further relaxed. On the other hand, since it has been observed [87] that this logarithmic-type of estimate cannot be avoided under any a priori assumption of type (0.2.52) for any finite $k=0,1,2, \ldots$, it seems natural to think that another direction to proceed would be the one of looking for different a priori assumptions rather than the one of type (0.2.52). For a complete analysis of open problems in this area we refer to [5].

\subsubsection{The anisotropic case}

\section{The non-uniqueness}

In anisotropic media the conductivity depends on the direction, therefore it is represented by a matrix $\gamma=\left(\gamma_{i j}\right)_{i, j=1}^{n}$, which is symmetric and positive definite. Anisotropic conductivity appears in nature, for example as a homogenization limit in layered or fibrous structures such as rock stratum or muscle, as a result of crystalline structure or of deformation of an isotropic material. Let $\Omega \subset \mathbb{R}^{n}$ be a domain with smooth boundary $\partial \Omega$ (a Lipschitz boundary will be enough in most cases). The Dirichlet problem associated in the anisotropic case takes the form

$$
\left\{\begin{array}{l}
\sum_{i, j=1}^{n} \frac{\partial}{\partial x^{i}}\left(\gamma_{i j} \frac{\partial u}{\partial x^{j}}\right)=0 \quad \text { in } \Omega \\
\left.u\right|_{\partial \Omega}=f
\end{array}\right.
$$

where $f \in H^{\frac{1}{2}}(\partial \Omega)$ is a prescribed potential at the boundary. The Dirichlet-toNeumann map associated with $\gamma$ is defined by

$$
\Lambda_{\gamma} f=\left.\gamma \nabla u \cdot \nu\right|_{\partial \Omega},
$$

for any u solution to (0.2.54). Here $\gamma \nabla u \cdot \nu=\left.\sum_{i, j=1}^{n}\left(\gamma_{i j} \frac{\partial u}{\partial x^{j}}\right) \nu_{i}\right|_{\partial \Omega}$ and as usual $\nu=\left(\nu_{i}\right)_{i=1}^{n}$ is the unit outer normal to $\partial \Omega$. The weak formulation of $(0.2 .55)$ is commonly used and will be given below for sake of completeness.

DEFINITION 0.2.1. The Dirichlet-to-Neumann map associated with (0.2.54) is

$$
\Lambda_{\gamma}: H^{\frac{1}{2}}(\partial \Omega) \longrightarrow H^{-\frac{1}{2}}(\partial \Omega)
$$


given by

$$
\left\langle\Lambda_{\gamma} f, \eta\right\rangle=\int_{\Omega} \sigma(x) \nabla u(x) \cdot \nabla \phi(x) d x,
$$

for any $f, \eta \in H^{\frac{1}{2}}(\partial \Omega), u, \phi \in H^{1}(\Omega),\left.\phi\right|_{\partial \Omega}=\eta$ and $u$ is the weak solution to $(0.2 .54)$.

A conductor is isotropic when $\gamma=\left(\gamma_{i j}\right)$ is rotation invariant, i.e., when at each point

$$
R^{T} \gamma R=\gamma,
$$

for all rotations $R$. This is the case exactly when $\gamma=\alpha I$, where $\alpha>0$ is a scalar function and $I$ the identity matrix.

We saw in section 0.2.1 that the uniqueness problem for the isotropic case can be considered solved; on the other hands, in the anisotropic case, $\Lambda_{\gamma}$ does not in general determine $\gamma$. Tartar (see [76]) observed the following non-uniqueness result.

PROPOSITION 0.2.14. If $\psi: \bar{\Omega} \longrightarrow \bar{\Omega}$ is a $C^{1}$ diffeomorphism such that $\psi(x)=x$, for each $x \in \partial \Omega$, then $\gamma$ and $\tilde{\gamma}=\frac{(D \psi) \gamma(D \gamma)^{T}}{\operatorname{det}(D \psi)} \circ \psi^{-1}$ have the same Dirichlet-to-Neumann map.

The proof of this result is given below as a tutorial for the first-time reader of this material.

Proof. Let us consider the change of variables $y=\psi(x)$ on the Dirichlet integral

$$
\int_{\Omega} \gamma_{i j}(x) \frac{\partial u}{\partial x^{i}} \frac{\partial u}{\partial x^{j}} d x=\int_{\Omega} \tilde{\gamma}_{i j}(y) \frac{\partial \tilde{u}}{\partial y^{i}} \frac{\partial \tilde{u}}{\partial y^{j}} d x
$$

where

$$
\tilde{\gamma}(y)=\frac{(D \psi) \gamma(D \psi)^{T}}{\operatorname{det}(D \psi)} \circ \psi^{-1}(y)
$$

and

$$
\tilde{u}(y)=u \circ \psi^{-1}(y) .
$$

Notice that the solution $u$ of the Dirichlet problem

$$
\left\{\begin{array}{l}
\nabla \cdot \gamma \nabla u=0 \quad \text { in } \Omega \\
\left.u\right|_{\partial \Omega}=f
\end{array}\right.
$$

minimizes the integral appearing on the left hand side of (0.2.57), therefore $\tilde{u}=u \circ \psi^{-1}$ minimizes the Dirichlet integral appearing on the right hand side of the same. One can then conclude that $\tilde{u}$ solves

$$
\left\{\begin{array}{l}
\nabla \cdot(\tilde{\gamma} \nabla \tilde{u})=0 \\
\left.\tilde{u}\right|_{\partial \Omega}=\tilde{f}=u \circ \psi^{-1} .
\end{array} \text { in } \Omega\right.
$$


Let us consider now the solution $v$ of

$$
\left\{\begin{array}{l}
\nabla \cdot(\gamma \nabla v)=0 \quad \text { in } \quad \Omega \\
\left.v\right|_{\partial \Omega}=g
\end{array}\right.
$$

and let $\tilde{v}$ be obtained by $v$ by the change of variable, therefore $\tilde{v}$ solves

$$
\left\{\begin{array}{l}
\nabla \cdot(\tilde{\gamma} \nabla \tilde{v})=0 \\
\left.\tilde{v}\right|_{\partial \Omega}=\tilde{g}=g \circ \psi^{-1} .
\end{array} \quad \text { in } \quad \Omega\right.
$$

By the change of variables in the Dirichlet integrals we get

$$
\int_{\Omega} \gamma_{i j} \frac{\partial u}{\partial x^{i}} \frac{\partial v}{\partial x^{j}} d x=\int_{\Omega} \tilde{\gamma}_{i j} \frac{\partial \tilde{u}}{\partial y^{i}} \frac{\partial \tilde{v}}{\partial y^{j}} d y
$$

which can be written as

$$
\int_{\Omega} \gamma \nabla u \cdot \nabla v d x=\int_{\Omega} \tilde{\gamma} \nabla \tilde{u} \cdot \nabla \tilde{v} d y
$$

which is equivalent to

$\int_{\Omega} \nabla \cdot(v \gamma \nabla u) d x-\int_{\Omega} v \nabla \cdot(\gamma \nabla u) d x=\int_{\Omega} \nabla \cdot(\tilde{v} \tilde{\gamma} \nabla \tilde{u}) d y-\int_{\Omega} \tilde{v} \nabla \cdot(\tilde{\gamma} \nabla \tilde{u}) d y$

and by the divergence theorem

$$
\int_{\partial \Omega} v \gamma \nabla u \cdot \nu d s=\int_{\partial \Omega} \tilde{v} \tilde{\gamma} \nabla \tilde{u} \cdot \nu d s
$$

but $\tilde{v}=v \circ \psi^{-1}=v=g$ and $\tilde{u}=u \circ \psi^{-1}=u=f$ at the boundary $\partial \Omega$, then

$$
\int_{\partial \Omega} g \Lambda_{\gamma}(f) d s=\int_{\partial \Omega} g \Lambda_{\tilde{\gamma}}(f) d s
$$

then $\Lambda_{\gamma}=\Lambda_{\tilde{\gamma}}$.

Since Tartar's observation has been made, different lines of research have been pursued. One direction was to prove the uniqueness of $\gamma$ up to diffeomorphisms that fix the boundary, whereas the other direction was to study conductivities with some a priori information. The first direction of research is summarized in what follows.

\section{Uniqueness up to diffeomorphism}

The question here is to investigate whether Tartar's observation is the only obstruction to unique identifiability of the conductivity. We start by observing that the physical problem of determining the conductivity of a body is closely related to the geometrical problem of determining a Riemannian metric from its Dirichlet-to-Neumann map for harmonics functions [82]. 
Let $(M, g)$ be a compact Riemannian manifold with boundary. The LaplaceBeltrami operator associated to the metric $g$ is given in local coordinates by

$$
\Delta_{g}:=\sum_{i j=1}^{n}(\operatorname{det} g)^{-\frac{1}{2}} \frac{\partial}{\partial x^{i}}\left\{(\operatorname{det} g)^{\frac{1}{2}} g^{i j} \frac{\partial u}{\partial x^{j}}\right\} .
$$

The Dirichlet-to-Neumann map associated to $g$ is the operator $\Lambda_{g}$ mapping functions $\left.u\right|_{\partial M} \in H^{1 / 2}(\partial M)$ into $(n-1)$-forms $\Lambda_{\sigma}\left(\left.u\right|_{\partial M}\right) \in H^{-1 / 2}\left(\Omega^{n-1}(\partial M)\right)$

$$
\Lambda_{g}(f)=i^{\star}\left(*_{g} d u\right)
$$

for any $u \in H^{1}(M)$ solution to $\Delta_{g} u=0$ in $M$, with $\left.u\right|_{\partial M}=f$. Here $i$ is the inclusion map $i: \partial M \rightarrow M$ and $i^{\star}$ denotes the pull-back of forms under the map $i$. In any local coordinates $(0.2 .58)$ becomes

$$
\Lambda_{g}(f)=\left.\sum_{i, j=1}^{n} \nu^{i} g^{i j} \frac{\partial u}{\partial x_{j}} \sqrt{\operatorname{det} g}\right|_{\partial M} .
$$

The inverse problem is to recover $g$ from $\Lambda_{g}$. In dimension $n \geq 3$, the conductivity $\gamma$ uniquely determines a Riemannian metric $g$ such that

$$
\gamma=*_{g}
$$

where $*_{g}$ is the Hodge operator associated the the metric $g$ mapping 1-forms on $M$ into $(n-1)$-forms (see [44], [81], [82]). In any local coordinates $(0.2 .60)$ becomes

$$
\left(g_{i j}\right)=\left(\operatorname{det} \gamma_{k l}\right)^{\frac{1}{n-2}}\left(\gamma_{i j}\right) \quad \text { and } \quad\left(\gamma_{i j}\right)=\left(\operatorname{det} g_{k l}\right)^{\frac{1}{2}}\left(g^{i j}\right),
$$

where $\left(g^{i j}\right),\left(\gamma^{i j}\right)$ denotes the matrix inverse of $\left(g_{i j}\right)$ and $\left(\gamma_{i j}\right)$ respectively. It has been shown in [82] that if $M$ is a domain in $\mathbb{R}^{n}$, then for $n \geq 3$

$$
\Lambda_{g}=\Lambda_{\gamma}
$$

In dimension $n \geq 3$ if $\psi$ is a diffeomorphism of $\bar{M}$ that fixes the boundary, we have

$$
\Lambda_{\psi^{*} g}=\Lambda_{g}
$$

where $\psi^{*} g$ is the pull-back of $g$ under $\psi$. For the case $n=2$ the situation is different as the two-dimensional conductivity determines a conformal structure of metrics under scalar field, i.e. there exists a metric $g$ such that $\gamma=\varphi *_{g}$, for a positive function $\varphi$. Therefore in $n=2$, if $\psi$ is a diffeomorphism of $\bar{M}$ that fixes the boundary, we have

$$
\Lambda_{\varphi \psi^{*} g}=\Lambda_{g},
$$

for any smooth positive function such that $\left.\varphi\right|_{\partial M}=1$. It seems natural to think that (0.2.63) and (0.2.64) are the only obstructions to uniqueness for $n \geq 3$ and $n=2$ respectively. In 1989 Lee and Uhlmann [82] formulated the following two conjectures. 
Conjecture 0.2.15. Let $\bar{M}$ be a smooth, compact n-manifold, with boundary, $n \geq 3$ and let $g, \tilde{g}$ be smooth Riemannian metrics on $\bar{M}$ such that

$$
\Lambda_{g}=\Lambda_{\tilde{g}}
$$

Then there exists a diffeomorphism $\psi: \bar{M} \rightarrow \bar{M}$ with $\left.\psi\right|_{\partial M}=I d$, such that $g=\psi^{\star} \tilde{g}$.

Conjecture 0.2.16. Let $\bar{M}$ be a smooth, compact 2-manifold with boundary, and let $g, \tilde{g}$ be smooth Riemannian metrics on $\bar{M}$ such that

$$
\Lambda_{g}=\Lambda_{\tilde{g}}
$$

Then there exists a diffeomorphism $\psi: \bar{M} \rightarrow \bar{M}$ with $\left.\psi\right|_{\partial M}=I d$, such that $\psi^{\star} \tilde{g}$ is a conformal multiple of $g$, in other words there exists $\phi \in C^{\infty}(\bar{M})$ such that

$$
\psi^{\star} \tilde{g}=\phi g .
$$

Conjecture 0.2.15 has been proved in [82] in a particular case. The result is the following.

THEOREM 0.2.17. Let $\bar{M}$ be a compact, connected, real-analytic n-manifold with connected real-analytic boundary, and assume that $\pi_{1}(\bar{M}, \partial M)=0$ (this assumption means that every closed path in $\bar{M}$ with base point in $\partial M$ is homotopic to some path that lies entirely in $\partial M)$. Let $g$ and $\tilde{g}$ be real-analytic metrics on $\bar{M}$ such that

$$
\Lambda_{g}=\Lambda_{\tilde{g}}
$$

and assume that one of the following conditions holds:

1. $\bar{M}$ is strongly convex with respect to both $g$ and $\tilde{g}$;

2. either $g$ or $\tilde{g}$ extends to a complete real-analytic metric on a non-compact real-analytic manifold $\tilde{M}$ (without boundary) containing $\bar{M}$.

Then there exists a real-analytic diffeomorphism $\psi: \bar{M} \rightarrow \bar{M}$ with $\left.\psi\right|_{\partial M}=I d$, such that $g=\psi^{\star} \tilde{g}$.

Theorem 0.2.17 has been proved by showing that one can recover the full Taylor series of the metric at the boundary from $\Lambda_{g}$. The diffeomorphism $\psi$ is then constructed by analytic continuation from the boundary. As we previously mentioned the full Taylor series of $\gamma$ was recovered by Kohn and Vogelius in [76] from the knowledge of $\Lambda_{\gamma}$ in the isotropic case and then a new proof was given in [108] by showing that the full symbol of the pseudodifferential operator $\Lambda_{\gamma}$ determines the full Taylor series of $\gamma$ at the boundary. In [82] a simpler method suggested by R. Melrose consisting of factorizing $\Delta_{g}$, is used. In 1990 Sylvester proved in [107] conjecture 0.2.16 in a particular case. His result is the following. 
THEOREM 0.2.18. Let $\Omega$ be a bounded domain in $\mathbb{R}^{2}$ with a $C^{3}$ boundary and let $\gamma_{1}, \gamma_{2}$ be anisotropic $C^{3}$ conductivities in $\bar{\Omega}$ such that

$$
\left\|\log \left(\operatorname{det} \gamma_{i}\right)\right\|_{C^{3}}<\varepsilon(M, \Omega), \quad \text { for } \quad i=1,2,
$$

with $M \geq\left\|\gamma_{i}\right\|_{C^{3}}$, for $i=1$, 2 and $\varepsilon(M, \Omega)$ sufficiently small. If

$$
\Lambda_{\gamma_{1}}=\Lambda_{\gamma_{2}},
$$

then there exists a $C^{3}$ diffeomorphism $\psi$ of $\bar{\Omega}$ such that $\left.\psi\right|_{\partial \Omega}=I d$ and such that

$$
\psi_{\star} \gamma_{1}=\gamma_{2} .
$$

Nachman [91] extended this result in 1995 by proving the same theorem but removing the hypothesis (0.2.65). In 1999 Lassas and Uhlmann [79] extended the result of [82]. They assumed that the Dirichlet-to-Neumann map is measured only on a part of the boundary which is assumed to be real-analytic in the case $n \geq 3$ and $C^{\infty}$-smooth in the two-dimensional case. The metric is here recovered (up to diffeomorphism) and the manifold is reconstructed. Since a manifold is a collection of coordinate patches, the idea is to construct a representative of an equivalent class of the set of isometric Riemannian manifolds $(M, g)$. Let us recall that if $\Gamma$ is an open subset of $\partial M$, we define

$$
\Lambda_{g, \Gamma}(f)=\left.\Lambda_{g}(f)\right|_{\Gamma},
$$

for any $f$ with supp $f \subseteq \Gamma$. The main result of [79] is given below.

THEOREM 0.2.19. Let us assume that one of the following conditions is satisfied:

1. $M$ is a connected Riemannian surface;

2. $n \geq 3$ and $(M, g)$ is a connected real-analytic Riemannian manifold and the boundary $\partial M$ is real-analytic in the non-empty set $\Gamma \subset \partial M$.

Then

1. For $\operatorname{dim} M=2$ the $\Lambda_{g, \Gamma-m a p p i n g}$ and $\Gamma$ determine the conformal class of the Riemannian manifold $(M, g)$.

2. For a real-analytic Riemannian manifold $(M, g)$, $\operatorname{dim} M>2$ which boundary is real analytic in $\Gamma$, the $\Lambda_{g}, \Gamma$-mapping and $\Gamma$ determine the Riemannian manifold $(M, g)$.

This result improved the one in [82] also because here the only assumption on the topology of the manifold is the connectedness, while in [82] the manifold was simply connected and the boundary of the manifold was assumed to be geodesically convex. Theorem 0.2 .19 has been extended in [80] to a completeness hypothesis on $\bar{M}$. 


\section{Anisotropy which is partially a priori known}

Another approach to the anisotropic problem is to assume that the conductivity $\gamma$ is a priori known to depend on a restricted number of unknown spatially dependent parameters. In 1984 Kohn and Vogelius (see [76]) considered the case where the conductivity matrix $\gamma=\left(\gamma_{i j}\right)$ is completely known with the exception of one eigenvalue. The main result is the following.

THEOREM 0.2.20. Let $\gamma, \tilde{\gamma}$ be two symmetric, positive definite matrices with entries in $L^{\infty}(\Omega)$, and let $\left\{\gamma_{i}\right\},\left\{\tilde{\gamma}_{i}\right\}$ and $\left\{e_{i}\right\},\left\{\tilde{e}_{i}\right\}$ be the corresponding eigenvalues and eigenvectors. For $x_{0} \in \partial \Omega$, let $B$ be a neighborhood of $x_{0}$ relative to $\bar{\Omega}$, and suppose that

$$
\begin{aligned}
& \gamma, \tilde{\gamma} \in C^{\infty}(B) ; \\
& \partial \Omega \cap B \quad \text { is } \quad C^{\infty} ; \\
& e_{j}=\tilde{e}_{j}, \quad \lambda_{j}=\tilde{\lambda}_{j} \quad \text { in } \quad B, \quad \text { for } \quad 1 \leq j \leq n-1 ; \\
& e_{n}\left(x_{0}\right) \cdot \nu\left(x_{0}\right) \neq 0 .
\end{aligned}
$$

If

$$
Q_{\gamma}(\phi)=Q_{\tilde{\gamma}}(\phi) \quad \text { for every } \quad \phi \in H^{\frac{1}{2}}(\partial \Omega)
$$

with supp $\phi \subset B \cap \partial \Omega$, then

$$
D^{k} \tilde{\lambda}_{n}\left(x_{0}\right)=D^{k} \lambda_{n}\left(x_{0}\right)
$$

for every $k=\left(k_{1}, \ldots, k_{n}\right), k_{i} \in \mathbb{Z}^{+}, i=1 \ldots n$.

In 1990 Alessandrini [3] considered the case in which $\gamma$ is a priori known to be of type

$$
\gamma(x)=A(a(x))
$$

where $t \rightarrow A(t)$ is a given matrix-valued function and $a=a(x)$ is an unknown scalar function. He proved results of uniqueness and stability at the boundary and then uniqueness in the interior among the class of piecewise real-analytic perturbations of the parameter $a(x)$. The main hypothesis he used is the socalled monotonicity assumption

$$
D_{t} A(t) \geq C I,
$$

where $C>0$ is a constant. In 1997 Lionheart [81] proved that the parameter $a(x)$ can be uniquely recovered for a conductivity $\gamma$ of type

$$
\gamma(x)=a(x) A_{0}(x),
$$

where $A_{0}(x)$ is given. Results in [3] have been extended in 2001 by Alessandrini and Gaburro [6] to a class of conductivities 


$$
\gamma(x)=A(x, a(x)),
$$

where $A(x, t)$ is given and satisfies the monotonicity condition with respect to the parameter $t$

$$
D_{t} A(x, t) \geq C I,
$$

where $C>0$ is a constant (see [6] or [43] for this argument). In [6] the authors improved results of [3] since they relaxed the hypothesis on $A(\cdot, t)$ for the global uniqueness in the interior and the result there obtained can be applied to [81] as well. The technique of [6] can also be applied to the so called one-eigenvalue problem introduced in [76]. Results of [6] have been recently extended to manifolds [45] and to the case when the local Dirichlet-to-Neumann map is prescribed on an open portion of the boundary [7].

\subsubsection{Some remarks on the Dirichlet-to-Neumann map EIT with partial data}

In many applications of EIT one can actually only take measurements of voltages and currents on some portion of the boundary. In such situation the Dirichletto-Neumann map can only be defined locally.

Let $\Omega \subseteq \mathbb{R}^{n}$ be a domain with conductivity $\gamma$. If $\Gamma$ is a non-empty open portion of $\partial \Omega$ we shall introduce the subspace of $H^{\frac{1}{2}}(\partial \Omega)$

$$
H_{c o}^{\frac{1}{2}}(\Gamma)=\left\{f \in H^{\frac{1}{2}}(\partial \Omega) \mid \operatorname{supp} f \subset \Gamma\right\} .
$$

DEFINITION 0.2.2. The local Dirichlet-to-Neumann map associated to $\gamma$ and $\Gamma$ is the operator

$$
\Lambda_{\gamma}^{\Gamma}: H_{c o}^{\frac{1}{2}}(\Gamma) \longrightarrow\left(H_{c o}^{\frac{1}{2}}(\Gamma)\right)^{*}
$$

defined by

$$
\left\langle\Lambda_{\gamma}^{\Gamma} f, \eta\right\rangle=\int_{\Omega} \gamma \nabla u \cdot \nabla \phi d x
$$

for any $f, \eta \in H_{c o}^{\frac{1}{2}}(\Gamma)$, where $u \in H^{1}(\Omega)$ is the weak solution to

$$
\begin{cases}\nabla \cdot(\gamma(x) \nabla u(x))=0, & \text { in } \Omega, \\ u=f, & \text { on } \partial \Omega,\end{cases}
$$

and $\phi \in H^{1}(\Omega)$ is any function such that $\left.\phi\right|_{\partial \Omega}=\eta$ in the trace sense.

Note that, by (0.2.72), it is easily verified that $\Lambda_{\sigma}^{\Gamma}$ is self adjoint. The inverse problem is to recover $\gamma$ from $\Lambda_{\gamma}^{\Gamma}$.

The procedure of reconstructing the conductivity by local measurements has been studied first by Brown [25], where the author gives a formula for reconstructing the isotropic conductivity pointwise at the boundary of a Lipschitz 
domain $\Omega$ without any a priori smoothness assumption of the conductivity. Nakamura and Tanuma [93] give a formula for the pointwise reconstruction of a conductivity continuous at one point $x^{0}$ of the boundary from the local D-N map when the boundary is $C^{1}$ near $x^{0}$. Under some additional regularity hypothesis the authors give a reconstruction formula for the normal derivatives of $\gamma$ on $\partial \Omega$ at $x^{0} \in \partial \Omega$ up to a certain order. A direct method for reconstructing the normal derivative of the conductivity from the local Dirichlet-to-Neumann (D-N) map is presented in [94]. The result in [93] has been improved by Kang and Yun [71] to an inductive reconstruction method by using only the value of $\gamma$ at $x^{0}$. The authors derive here also Hölder stability estimates for the inverse problem to identify Riemannian metrics (up to isometry) on the boundary via the local D-N map. An overview on reconstructing formulas of the conductivity and its normal derivative can be found in [95].

For related uniqueness results in the case of local boundary data, we refer to Alessandrini and Gaburro [7], Bukhgeim and Uhlmann [27], Kenig, Sjöstrand and Uhlmann [74] and Isakov [69], and, for stability, [7] and Heck and Wang [57]. We refer also to the more recents results of Kenig-Salo ([72], [73]). We would like to stress out that [73] generalizes the results obtained in both [74] and [69] by making use of improved Carleman estimates with boundary terms, complex geometrical optics solutions involving reflected Gaussian beam quasimodes and invertibility of (broken) geodesics ray transforms. Results of stability for cases of piecewise constant conductivities and local boundary maps have also been obtained by Alessandrini and Vessella [8], and by Di Cristo [36]. We also refer to $[112$, section 7$]$.

\section{The Neumann-to-Dirichlet map}

In many applications of EIT especially in medical imaging, rather than the local Dirichlet-to-Neumann map, one should consider the so-called local Neumann-toDirichlet (N-D) map. That is, the map associating to specified current densities supported on a portion $\Gamma \subset \partial \Omega$ the corresponding boundary voltages, also measured on the same portion $\Gamma$ of $\partial \Omega$. Usually electrodes are only applied to part of the body and in geophysics of course we have an extreme example where $\Gamma$ is a small portion of the surface of the earth $\Omega$. It seems appropriate at this stage to recall the definition of the N-D map and its local version for sake of completeness [7].

Let us introduce the following function spaces (see [7])

$$
\begin{aligned}
H_{\diamond}^{\frac{1}{2}}(\partial \Omega) & =\left\{\phi \in H^{\frac{1}{2}}(\partial \Omega) \mid \int_{\partial \Omega} \phi=0\right\}, \\
H_{\diamond}^{-\frac{1}{2}}(\partial \Omega) & =\left\{\psi \in H^{-\frac{1}{2}}(\partial \Omega) \mid\langle\psi, 1\rangle=0\right\} .
\end{aligned}
$$

Observe that if we consider the (global) D-N map $\Lambda_{\gamma}$, that is the map introduced in (0.2.71) $\Lambda_{\gamma}^{\Gamma}$ in the special case when $\Gamma=\partial \Omega$, we have that, it maps onto ${ }_{0} H^{-\frac{1}{2}}(\partial \Omega)$, and, when restricted to $H_{\diamond}^{\frac{1}{2}}(\partial \Omega)$, it is injective with bounded inverse. Then we can define the global Neumann-to-Dirichlet map as follows. 
DEFINITION 0.2.3. The Neumann-to-Dirichlet map associated to $\gamma, N_{\gamma}$ : $H_{\diamond}^{-\frac{1}{2}}(\partial \Omega) \longrightarrow H_{\diamond}^{\frac{1}{2}}(\partial \Omega)$ is given by

$$
N_{\gamma}=\left(\left.\Lambda_{\gamma}\right|_{0 H^{\frac{1}{2}}(\partial \Omega)}\right)^{-1}
$$

Note that $N_{\gamma}$ can also be characterized as the self adjoint operator satisfying

$$
\left\langle\psi, N_{\gamma} \psi\right\rangle=\int_{\Omega} \gamma(x) \nabla u(x) \cdot \nabla u(x) d x
$$

for every $\psi \in H_{\diamond}^{-\frac{1}{2}}(\partial \Omega)$, where $u \in H^{1}(\Omega)$ is the weak solution to the Neumann problem

$$
\begin{cases}L_{\gamma} u=0, & \text { in } \Omega, \\ \left.\gamma \nabla u \cdot \nu\right|_{\partial \Omega}=\psi, & \text { on } \partial \Omega \\ \int_{\partial \Omega} u=0 . & \end{cases}
$$

We are now in position to introduce the local version of the N-D map. Let $\Gamma$ be an open portion of $\partial \Omega$ and let $\Delta=\partial \Omega \backslash \bar{\Gamma}$. We denote by $H_{00}^{\frac{1}{2}}(\Delta)$ the closure in $H^{\frac{1}{2}}(\partial \Omega)$ of the space $H_{c o}^{\frac{1}{2}}(\Delta)$ previously defined in (0.2.70) and we introduce

$$
H_{\diamond}^{-\frac{1}{2}}(\Gamma)=\left\{\psi \in H_{\diamond}^{-\frac{1}{2}}(\partial \Omega) \mid\langle\psi, f\rangle=0, \quad \text { for any } f \in H_{00}^{\frac{1}{2}}(\Delta)\right\},
$$

that is the space of distributions $\psi \in H^{-\frac{1}{2}}(\partial \Omega)$ which are supported in $\bar{\Gamma}$ and have zero average on $\partial \Omega$. The local N-D map is then defined as follows.

DEFINITION 0.2.4. The local Neumann-to-Dirichlet map associated to $\gamma, \Gamma$ is the operator $N_{\gamma}^{\Gamma}:{ }_{0} H^{-\frac{1}{2}}(\Gamma) \longrightarrow\left({ }_{0} H^{-\frac{1}{2}}(\Gamma)\right)^{\star} \subset{ }_{0} H^{\frac{1}{2}}(\partial \Omega)$ given by

$$
\left\langle N_{\gamma}^{\Gamma} i, j\right\rangle=\left\langle N_{\gamma} i, j\right\rangle
$$

for every $i, j \in{ }_{0} H^{-\frac{1}{2}}(\Gamma)$.

\subsection{The Reconstruction problem}

\subsubsection{Locating objects and boundaries}

The simplest form of the inverse problem is to locate a single object with a conductivity contrast in a homogeneous medium. Some real situations approximate this, such as a weakly electric fish locating a single prey or the location of an insulating land mine in homogeneous soil. Typically the first test done on an EIT system experimentally is to locate a cylindrical or spherical object in a cylindrical tank. Linearization about $\gamma=1$ simplifies to

$$
\nabla^{2} w=-\nabla \delta \cdot \nabla u+O\left(\|\delta\|_{L^{\infty}}^{2}\right) .
$$

We see the disturbance in the potential $w$ is, to first order, the solution of Poisson's equation with a dipole source centred on the object oriented in the 
direction of the unperturbed electric field. With practice experienced experimenters (like electric fish) can roughly locate the object from looking at a display of the voltage changes. When it is known a priori that there is single object, either small or with a known shape, the reconstruction problem is simply fitting a small number of model parameters (eg position, diameter, conductivity contrast) to the measured voltage data, using an analytical or numerical forward solver. This can be achieved using standard nonlinear optimization methods. For the two dimensional case a fast mathematically rigorous method of locating an object (not required to be circular) from one set of Cauchy data is presented by Hanke [51]. Results on the recovery of the support of the difference between two piece-wise analytic conductivities by solving the linearized problem is given in $[55]$.

In the limiting case where the object is insulating, object location becomes a free boundary problem, where the Dirichlet to Neumann map is known on the known boundary and only zero Neumann data known on the unknown boundary. This is treated theoretically for example by [62] and numerically in [63]. A practical example is the location of the air core of a hydrocyclone, a device used in chemical engineering [117].

In more complex cases the conductivity may be piecewise constant with a jump discontinuity on a smooth surface. In that case there are several methods that have been tested at least on laboratory data for locating the surface of the discontinuity. One would expect in general that the location of a surface can be achieved more accurately or with less data than the recovery of a spatially varying function and this is confirmed by numerical studies.

A natural method of representing the surface of discontinuity is as a level set of a smooth surface. This approach has the advantage that no change in parameterisation is required as the number of connected components changes, in contrast for example to representing a number of star-shaped objects using spherical polar coordinates. The approach to using the level set method in EIT is exactly the same as its use in scattering problems apart from the forward problem. Level set methods have been tested on experimental ERT and ECT data by Soleimani et al [104] and we reproduce some of their results in Figure 5 and we use some of their results in Figure 3.

Another approach to locating a discontinuity, common with other inverse boundary value problems for PDEs are "sampling and probe methods" in which a test is performed at each point in a grid to determine if that point is in the object. Linear sampling and factorization methods are treated in Chapter Theory and numerical results for the application of Linear Sampling to ERT for a half space are given by Hanke and Schappel[53]. Sampling methods generally require the the complete transfer impedance matrix and where only incomplete measurements are available they must be interpolated.

Also in the spirit of probe methods is the monotonicity method of Tamburrino and Rubinacci [111]. This method follows from the observation that for $\gamma$ real the map $\gamma \mapsto \mathbf{Z}_{\gamma}$ is monotone in the sense that $\gamma_{1} \leq \gamma_{2} \Rightarrow \mathbf{Z}_{\gamma_{1}}-\mathbf{Z}_{\gamma_{2}} \geq 0$, where a matrix $Z \geq 0$ if its eigenvalues are non-negative. Suppose that for some 


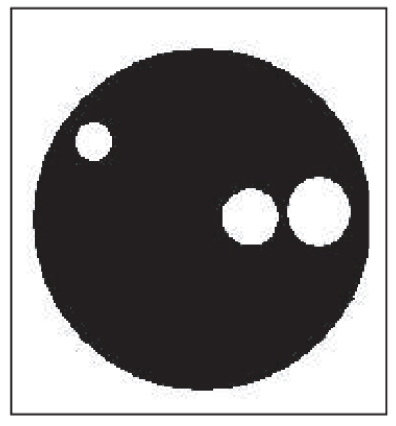

(a)

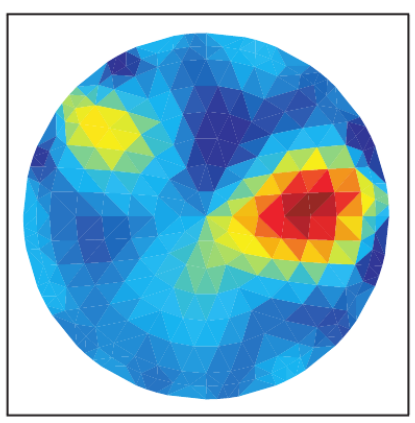

(b)

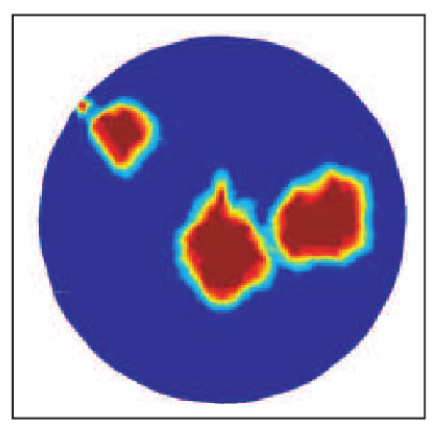

(c)

(a) Level set reconstruction (c) from experimental ERT data for high contrast objects (a) compared with generalized Tikhonov regularization.

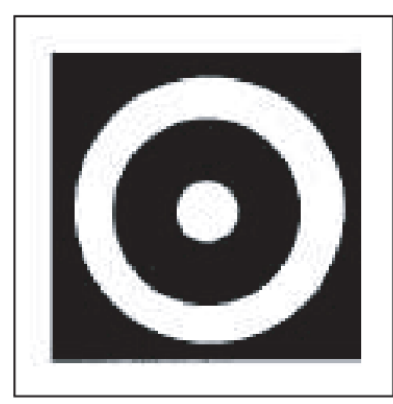

(a)

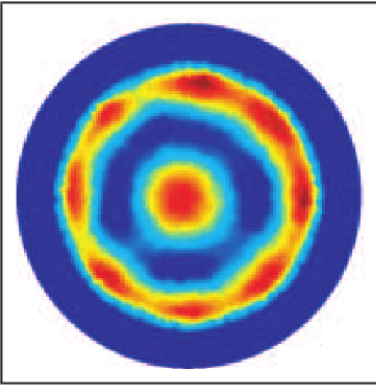

(b)

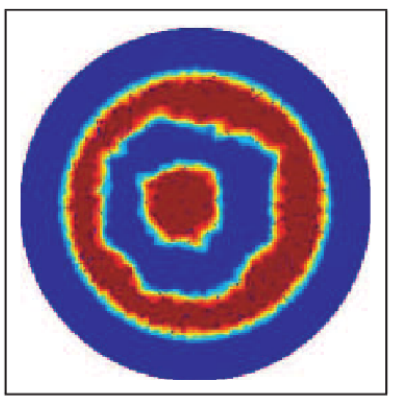

(c)

(b) Level set reconstruction (c) from experimental ECT data for a pipe (a) compared with generalized Tikhonov regularization.

Figure 5: Comparison of level set reconstruction of 2D experimental data compared to generalized Tikhonov regularization using a Laplacian smoothing matrix (EIDORS-2D [113]). Thanks to Manuchehr Soleimani for reconstruction results, and Wu Quaing Yang and colleagues for the ECT data[118], the experimental ERT data was from [113]. 
partition $\left\{\Omega_{i}\right\}$ of $\Omega$ (for example pixels or voxels)

$$
\gamma=\sum_{i} \gamma_{i} \chi_{\Omega_{i}}
$$

and each $\gamma_{i} \in\{m, M\}, 0<m<M$. For each $i$ let $\mathbf{Z}_{i}^{m}$ be the transfer impedance for a conductivity that is $M$ on $\Omega_{i}$ and $m$ elsewhere. Now suppose $\mathbf{Z}-\mathbf{Z}_{i}^{m}$ has a negative eigenvalue, then we know $\gamma_{i}=m$. For each set in the partition the test is repeated, and it is inferred that some of the conductivity values are definitely $m$, the equivalent procedure is repeated for each $\mathbf{Z}_{i}^{M}$. In practice, for large enough sets in the partition and $M-m$ big enough, most conductivity values in the binary image are determined, although this is not guaranteed. In practice the method is very fast as $\mathbf{Z}_{i}^{m}$ and $\mathbf{Z}_{i}^{M}$ can be precomputed and one only needs to find the smallest eigenvalue of two modestly sized matrices for each set in the partition. In the presence of noise, of course, one needs a sufficiently negative eigenvalue to be sure of the result of the test, and the method does assume that the conductivity is of the given form (0.3.2). Recently a partial converse of the monotonicity result has been found [?] and this promises more accurate fast methods of reconstructing the shape of an inclusion.

If conductivies on some sets are undetermined they can then be found using other methods. For example [116] use a Markov Chain Monte Carlo method to determine the expected value and variance of undetermined pixels in ECT.

\subsubsection{Forward solution}

Most reconstruction algorithms for EIT necessitate solution of the forward problem, that is to predict the boundary data given the conductivity. In addition, methods that use linearisation typically require electric fields in the interior. The simplest case is an algorithm that uses a linear approximation calculated at a homogenous background conductivity. For simple geometries this might be done using an analytical method, while for arbitrary boundaries Boundary Element Method is a good choice, and is also suitable for the case where the conductivity is piecewise constant with discontinuities on smooth surfaces. For general conductivities the choice is between finite difference, finite volume and finite element methods. All have been used in EIT problems. Finite element method (FEM) has the advantage that the mesh can be adapted to a general boundary surface and to the shape and location of electrodes, whereas regular grids in finite difference/volume methods can result in more efficient computation, traded off against the fine discretization needed to represent irregular boundaries. One could also use a hybrid method such as finite element on a bounded domain of variable conductivity coupled to BEM for a homogeneous (possibly unbounded) domain.

In reconstruction methods that iteratively adjust the conductivity and resolve the forward problem, a fast forward solution is needed, whereas in methods using a linear approximation, the forward solution can be solved off-line and speed is much less important. 
The simplest, and currently in EIT the most widely used, FE method is first order tetrahedral elements. Here a polyhedral approximation $\Omega_{h}$ to $\Omega$ is partitioned in to a finite set of tetrahedra $T_{k}, k=1, \ldots, n_{t}$ which overlap at most in a shared face, and with vertices $x_{i}, i=1<\ldots n_{v}$. The potential is approximated as a sum

$$
u_{h}(x)=\sum u_{i} \phi_{i}(x)
$$

where the $\phi_{i}$ are piecewise linear continuous functions with $\phi_{i}\left(x_{j}\right)=\delta_{i j}$. The finite element system matrix $K \in \mathbb{C}^{n_{v} \times n_{v}}$ is given by

$$
K_{i j}=\int_{\Omega_{p}} \gamma \nabla \phi_{i} \cdot \nabla \phi_{j} d x
$$

On each tetrahedron $\nabla \phi_{i}$ is constant which reduces calculation of (0.3.4) in the isotropic case to the mean of $\gamma$ on each tetrahedron. One then chooses an approximation to the conductivity in some space spanned by basis functions $\psi_{i}(x)$

$$
\gamma=\sum_{i} \gamma_{i} \psi_{i}
$$

One can choose these functions to implement some a priori constraints such as smoothness and to reduce the number of unknowns in the discrete inverse problem. Or one can choose basis functions just as the characteristic functions of the tetrahedra, which makes the calculation, and updating, of the system matrix very simple. In this case, all a priori information must be incorporated later, such as by a regulariziation term. In general the integrals

$$
\int_{\Omega_{p}} \psi_{l} \nabla \phi_{i} \cdot \nabla \phi_{j} d x .
$$

are evaluated using quadrature if they cannot be done explicitly. If the inverse solution uses repeated forward solutions with updated conductivity but with a fixed mesh, the coefficients (0.3.6) can be calculated once for each mesh and stored. For a boundary current density $j=\gamma \nabla u \cdot \nu$ we define the current vector $\mathbf{Q} \in \mathbb{R}^{n_{v}}$ by

$$
q_{i}=\int_{\partial \Omega} j \phi_{i} d x
$$

and the FE system is

$$
\mathbf{K u}=\mathbf{Q}
$$

where $\mathbf{u}$ is the vector of $u_{i}$. One additional condition is required for a unique solution, as the voltage is only determined up to an additive constant. One way to do this is to choose one ("grounded") vertex $i_{g}$ and enforce $u_{i_{g}}=0$ by deleting the $i_{g}$ row and column from the system (0.3.8). It is clear from (0.3.4) that for a pair of vertices indexed by $i, j$ that are not both in any tetrahedron, $K_{i j}=0$. The system (0.3.8) is equivalent to Ohm's and Kirchoff's law for a 
resistor network with resistors connecting nodes $i$ and $j$ when the corresponding vertices in the $\mathrm{FE}$ mesh share and edge (where some dihedral angles are obtuse we must allow the possibility of negative conductances). It is worth noting that whatever basis is user to represent the approximate conductivity (including an anisotropic conductivity) the finite element system has only one degree of freedom per edge and we cannot hope, even with perfect data and arithmetic, to recover more than $n_{e}$ (the number of edges) unknowns from our discretization of the inverse problem.

The above formulation implements the shunt model. The Complete Electrode Model (CEM) with specified currents can be implemented following Vauhkonen[114] using an augmented matrix. We define

$$
K_{i j}^{\circ}=K_{i j}+\sum_{l=1}^{L} \frac{1}{z_{l}} \int_{E_{l}} \phi_{i} \phi_{j} d x,
$$

where, here, $\left|E_{l}\right|$ denotes the area of the $l$ th electrode, and

$$
\begin{gathered}
K_{\ell \ell}^{\partial}=\frac{1}{z_{\ell}}\left|E_{\ell}\right| \quad \text { for } \quad \ell=1, \ldots, L, \\
K_{i \ell}^{\circ \partial}=-\int_{E_{l} l} \frac{1}{z_{\ell}} \phi_{i} d x \quad i=1, . ., n, \ell=1, . ., L
\end{gathered}
$$

The system matrix for the CEM, $\mathbf{K}^{\mathrm{CEM}} \in \mathbb{C}^{\left(n_{v}+L\right) \times\left(n_{v}+L\right)}$ is

$$
\mathbf{K}^{\mathrm{CEM}}=\left[\begin{array}{cc}
K^{\circ} & K^{\circ \partial} \\
K^{\circ \partial T} & K^{\partial}
\end{array}\right]
$$

In this notation, the linear system of equations has the form

$$
K^{\mathrm{CEM}} \tilde{\mathbf{u}}=\tilde{\mathbf{Q}}
$$

where $\tilde{\mathbf{u}}=\left(u_{1}, \ldots, u_{n_{v}}, V_{1}, . . V_{L}\right)^{T}$ and $\tilde{\mathbf{Q}}=\left(0, \ldots, 0, I_{1}, \ldots, I_{L}\right)^{T}$. The constraint $\mathbf{V} \in S$ (see section.0.1.3) is often used to ensure uniqueness of solution. The transfer impedance matrix is obtained directly as

$$
\mathbf{Z}=\left(K^{\partial}-K^{\circ \partial T} K^{\circ \dagger} K^{\circ \partial}\right)^{\dagger}
$$

although it is usual to solve the system 0.3 .12 as $u$ in the interior is used in the calculation of the linearization. This formulation should only be used for reasonably large $z_{\ell}$, as small $z_{\ell}$ will result in the block $K^{\partial}$ dominating the matrix. For an accurate forward model it is necessary to estimate the contact impedance accurately. This is more important when measurements from current carrying electrodes are used in the reconstruction, or when the electrodes are large (even if they are "passive" $I_{\ell}=0$ ). The CEM boundary condition is rather unusual and most commercial FE systems will not include the boundary condition easily. This is one of the reasons forward solution code for EIT is generally written specifically for the purpose, such as the EIDORS project[1]. 
It is possible to calculate the transadmitance matrix $\mathbf{Y}=\mathbf{Z}^{\dagger}$ more easily with standard solvers. One sets Robin boundary $u+z_{\ell} \gamma \partial u / \partial \nu=V_{\ell}$ on each $E_{\ell}$ and the zero Neumann condition (0.1.4) using $\mathbf{V}$ forming a basis for $S$, one then takes the integral of the current over each electrode as the current $\mathbf{I}=\mathbf{Y} \mathbf{V}$. For a given current pattern $\mathbf{I}$ one applies the Robin conditions $\mathbf{V}=\mathbf{Y}^{\dagger} \mathbf{I}$ and the solver gives the correct $u$. Advantages of commercial solver are that they might contain a wide variety of element types, fast solvers and mesh generators. Disadvantages are that they may be hard to integrate as part of a non-linear inverse solver, and it might be harder to calculate the linearization efficiently.

In fact implementing code to assemble a system matrix is quite straightforward; much harder for EIT is the generation of three dimensional meshes. For human bodies with irregular boundaries of inaccurately known shape this is a major problem. To apply boundary conditions accurately without overfine meshes it is also important that the electrodes are approximated by unions of faces of the elements. While the accuracy of the finite element method is well understood in terms of the error in the solution $u$, in EIT we require that the dependence of the boundary data on the conductivity is accurate, something that is not so well understood. In addition if the conductivities vary widely it may be necessary to remesh to obtain the required accuracy, and ideally this capability with be integrated with the inverse solver [89].

\subsubsection{Regularized linear methods}

Methods based on linearization are popular in medical and process versions of EIT. The reasons are twofold. Process and medical applications benefit from very rapid data acquisition times with even early systems capable measuring a transfer impedance matrix in less that $0.04 \mathrm{~s}$, and it was often required to produce an image in real time. The application of a precomputed (reguarized) inverse of the linearized forward problem required only about $\frac{1}{2} L^{2}(L-1)^{2}$ floating point operations. For reasons of both speed and economy early systems also assumed a two dimensional object with a single ring of electrodes arranged in a plane. The second reason for using a linear approximation is that in medical applications especially there is uncertainty in the body shape, electrode position and contact impedance. This means that a computed forward solution, based on an assumed conductivity (typically constant), has a much larger error than the errors inherent in the measurements. A compromise called difference imaging (by contrast to absolute imaging) uses a forward solution to calculate the linearization (0.2.8) and then forms an image of the difference of the conductivities between two different times, for example inspiration and expiration in a study of the lungs. Alternatively measurements can be taken simultaneously at two frequencies and a difference image formed of the permittivity.

Given a basis of applied current patterns $\mathbf{I}_{i}$ and a chosen set of measurements $\mathbf{M}_{i}$ expressed as a set of independent vectors in $S$ that are $1 /\left|E_{l}\right|$ for one electrode $E_{l},-1 /\left|E_{k}\right|$ for another electrode $E_{k}$ (the two electrodes between which we measure the voltage), and a set of functions $\psi_{i}$ with our approximate admittivity satisfying $\tilde{\gamma}=\sum \gamma_{k} \psi_{k}$, the discretization of the Fréchet derivative 
is the Jacobian matrix

$$
J_{(i j) k}=\frac{\partial}{\partial \gamma_{k}} \mathrm{M}_{i}^{T} \mathrm{ZI}_{j}=-\int_{\Omega} \psi_{k} \nabla v_{i} \cdot \nabla u_{j} d x
$$

where $L_{\tilde{\gamma}} u_{i}=L_{\tilde{\gamma}} v_{j}=0$ (at least approximately) with $u_{i}$ satisfying the CEM with current $\mathrm{I}_{i}$ and $v_{j}$ with $\mathrm{M}_{j}$. If the finite element approximation is used to solve the forward problem it has the interesting feature that the natural approximation to the Fréchet derivative in the FE context coincides with the Fréchet derivative of them FE approximation. The indices $(i j)$ are bracketed together as they are typically 'flattened' so the matrix of measurements becomes a vector and $J$ a matrix (rather than a tensor). Let $\tilde{\mathbf{V}}$ be the vector of all voltage measurements, $\tilde{\mathbf{V}}_{\text {calc }}$ the calculated voltages, and $\gamma$ the vector of $\gamma_{i}$. Our regularized least-squares version of the linearized problem is now

$$
\boldsymbol{\gamma}_{\text {reg }}=\underset{\gamma}{\arg \min }\left\|\mathbf{J} \boldsymbol{\gamma}-\left(\tilde{\mathbf{V}}-\tilde{\mathbf{V}}_{\text {calc }}\right)\right\|^{2}+\alpha^{2} \Psi(\boldsymbol{\gamma})
$$

where $\Psi$ is a penalty function and $\alpha$ a regularization parameter. The same formulation is used for difference imaging where $\tilde{\mathbf{V}}_{\text {calc }}$ is replaced by measured data at a different time or frequency. Typical choices for $\Psi$ are quadratic penalties such a weighted sum of squares of the $\gamma_{i}$, the two norm of (a discretization) a partial differential operator $\mathbf{R}$ applied to $\gamma-\gamma_{0}$, for some assumed background $\gamma_{0} \cdot\left\|\mathbf{R}\left(\gamma-\gamma_{0}\right)\right\|^{2}$. Another common choice is a weighted sum of squares, ie $L$ a positive diagonal matrix. In Total Variation regularization $\Psi$ approximates $\left\|\nabla\left(\gamma-\gamma_{0}\right)\right\|_{1}$, and can be used where discontinuities are expected in the conductivity. Where there is a jump discontinuity on a surface (a curve in the two-dimensional case) the total variation is the integral of the absolute value of the jump over the surface (curve). The singular values of $J$ are found to decay faster than exponentially (see fig 9), so it is a severely illconditioned problem and regularization is needed even for very accurate data. There are also to some extent diminishing returns in increasing the number of electrodes without also increasing the measurement accuracy.

For a quadratic penalty function the minimization problem with $\Psi(\gamma)=$ $\left\|\mathbf{R}\left(\gamma-\gamma_{0}\right)\right\|^{2}$ the solution to $(0.3 .16)$ is given by the well-known Tikhonov inversion formula

$$
\gamma_{\text {reg }}-\gamma_{0}=\left(\mathbf{J}^{*} \mathbf{J}+\alpha^{2} \mathbf{R}^{*} \mathbf{R}\right)^{-1} \mathbf{J}^{*}\left(\tilde{\mathbf{V}}-\tilde{\mathbf{V}}_{\text {calc }}\right)
$$

For a total variation penalty $\Psi(\gamma)=\left\|\mathbf{R}\left(\gamma-\gamma_{0}\right)\right\|_{1}$ minimization is more difficult, and standard gradient based optimization methods have difficulty with the singularity in $\Psi$ where a component of $\mathbf{R} \boldsymbol{\gamma}$ vanishes. One way around this is to use the Primal Dual Interior Point Method; for details, see [18] and for comparison of TV and a quadratic penalty applied to a difference image of the chest, see fig 6 .

\subsubsection{Regularized iterative non-linear methods}

As the problem is non-linear clearly a solution based on linearization is inaccurate. Intuitively there are two aspects to the non-linearity that are lost in a 


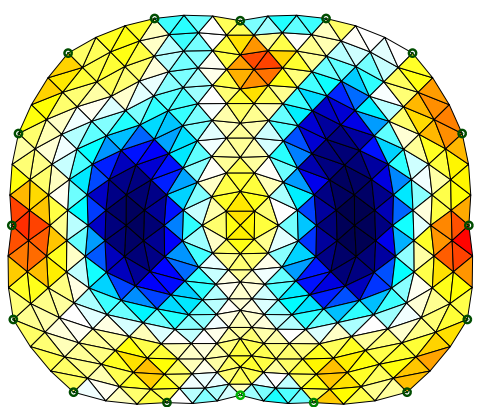

(a) Generalized Tikhonov

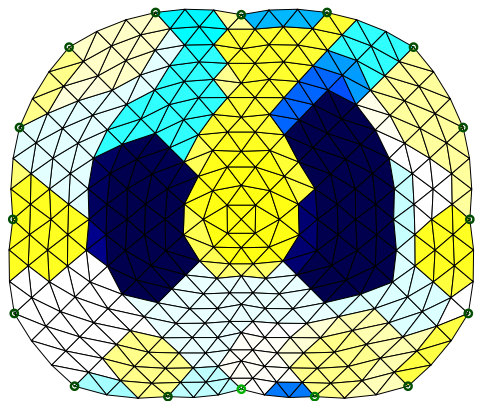

(b) Total variation

Figure 6: Time difference EIT image of a human thorax during breathing, comparison of generalized Tikhonov $\|\mathbf{R} \gamma\|_{2}^{2}$ and of the TV $\|\mathbf{R} \gamma\|_{1}$ regularized algorithms. Both are represented on the same colour scale and in arbitrary conductivity units. See [18] for details.
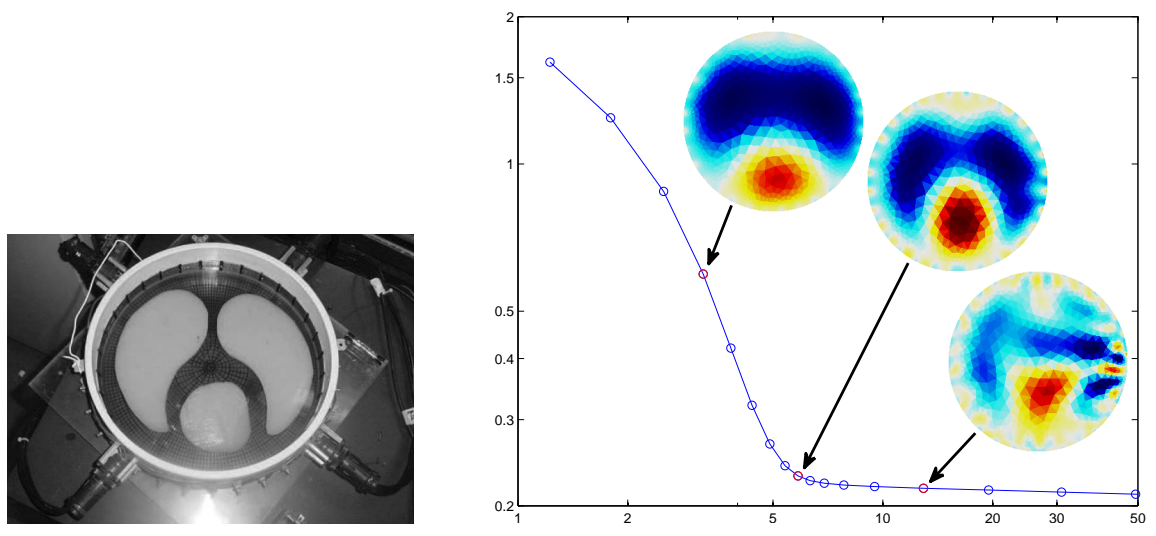

Figure 7: An "L-curve": data mismatch $\left\|\mathbf{V}-\mathbf{V}_{\text {calc }}(\gamma)\right\|_{2}$ (vertical) versus regularization norm $\left\|\mathbf{R}\left(\gamma-\gamma_{0}\right)\right\|_{2}$ (horizontal) for a range of 6 orders of magnitude of the regularization. In each case, a single step of the iterative solution was taken. Three representative images are shown illustrating the "overregularization", appropriate regularization, and "underregularization". The data are from the RPI chest phantom [66] shown left. 
linear approximation. If one considers an object of constant conductivity away from the boundary the norm of the voltage data will exhibit a sigmoid curve as the conductivity of that object varies, as seen in the example of a concentric anomaly and illustrated numerically in fig 10 . This means that voltage measurements saturate, or tends to a limiting value, as the conductivity contrast to the background tends to zero or infinity. Typically this means that linear approximations underestimate conductivity contrast. One has to be carefull in communications between mathematicians and engineers: the latter will sometimes take linearity (eg of $\mathbf{Y}(\gamma)$ ) to mean a function that is homogenous of degree one, ignoring the requirement for "superposition of solutions". If we consider two small spherical objects in a homogeneous background we know from (0.2.5) that to first order the change in $u$ due to the objects is approximately the sum of two dipole fields. The effect of non-linearity, the higher order terms in (0.2.5) can be thought of as interference between these two fields, analogous to higher order scattering in wave scattering problems. The practical effect is that linear approximations are not only poor at getting the correct conductivity contrast, but also poor at resolving a region between two objects that are close together. Many non-linear solution methods calculate an update of the admittivity from solving a linear system, that update is applied to the conductivity in the model and the forward solution solved again. One severe problem with linear reconstruction methods that do not include a forward solver is that one cannot test if the updated admittivity even fits the data better than the initial assumption (for example of a constant admittivity). Such algorithms tend to produce some plausible image even if the data are erroneous.

The usual approach taken in geophysical and medical EIT to nonlinear reconstruction is to numerically perform the (non-linear generalized Tikhonov) minimization

$$
\gamma_{\text {reg }}=\underset{\gamma}{\arg \min }\left\|\tilde{\mathbf{V}}_{\text {calc }}(\gamma)-\mathbf{V}\right\|^{2}+\alpha^{2} \Psi(\gamma)
$$

using standard numerical optimization techniques. As the Jacobian is known explicitly it is efficient to use gradient optimization methods such as GaussNewton, and in that context the update step is very similar to the solution of the linear problem (0.3.16), and is a linear system for quadratic $\Psi$. Assuming conductivity initialized as the background level $\gamma_{0}$ a typical iterative update scheme for successive approximations to the conductivity is

$$
\boldsymbol{\gamma}_{n+1}=\boldsymbol{\gamma}_{n}+\left(\mathbf{J}_{n}^{*} \mathbf{J}_{n}+\alpha^{2} \mathbf{R}^{*} \mathbf{R}\right)^{-1}\left(\mathbf{J}_{n}^{*}\left(\tilde{\mathbf{V}}-\tilde{\mathbf{V}}_{\text {calc }}\left(\boldsymbol{\gamma}_{n}\right)+\alpha^{2} \mathbf{R}^{*} \mathbf{R}\left(\boldsymbol{\gamma}_{0}-\boldsymbol{\gamma}_{n}\right)\right)\right.
$$

In contrast to the linearized problem, the non-linear problem requires repeated solution of the forward solution $\tilde{\mathbf{V}}_{\text {calc }}\left(\boldsymbol{\gamma}_{n}\right)$ for variable conductivity, typically using the finite element or finite difference method. One also has to constrain the conductivity $\operatorname{Re} \gamma$ to be positive and this is made easier by a choice of $\phi_{i}$ as the characteristic functions of a partition on $\Omega$. This could be a rectangular grid or a courser tetrahedral mesh than that used for $u$. Accurate modelling of electrodes requires a fine discretization near electrodes, and yet one cannot hope to recover that level of detail in the admittivity near an electrode. In 
many practical situations a priori bounds are known for the conductivity and permittivity and as the logarithmic stability result predicts, enforcing these bounds has a stabilising effect on the reconstruction. The positivity constraint can be enforced by a change of variables to $\log \gamma$ and this is common practice, with the Jacobian adjusted accordingly. It is generally better to perform a line search in the update direction from (0.3.19) to minimize the cost function in (0.3.18) rather than simply applying the update. Mostly commonly this search is approximated, for example by fitting a few points to a low order polynomial although implementation details of this are rarely well documented. It is also worth mentioning that most absolute reconstruction algorithms start by finding a homogeneous conductivity $\gamma_{0}$ best fitting the data before the iterative method starts.

In geophysical ERT nonlinear solution is well-established. Although it is more common, for reasons of economy, to measure only along a line and reconstruct on the plane beneath that line, fully three dimensional reconstruction is also widely used. The most common reconstruction code used is RES3DINV[46] which builds on the work of Loke and Barker at the University of Birmingham[85]. The code is available commercially from Loke's company Geotomo Software. RES3DINV has a finite difference forward solver used when the ground is assumed flat, and a finite element solver for known non-flat topography. In geophysical applications there is the advantage that obtaining a trangularization of the surface is common surveying practice. The Jacobian is intialised using an analytical initial solution assuming homogeneous conductivity. Regularized non-linear inversion is performed using Gauss-Newton, with recalculation of Jacobian[46], or using a quasi-Newton method in which a rank one update is performed on the Jacobian. The penalty function used in the regularization is of the form $\Psi(\gamma)=\|\mathbf{R} \gamma\|_{2}^{2}$ where $\mathbf{R}$ is an approximate differential operator that penalises horizontal and vertical variations differently. Total variation regularization $\Psi(\gamma)=\|\mathbf{R} \gamma\|_{1}$ is also an option in this code. When data is likely to be noisy one can select one can select a "robust error norm", in which the one-norm is used also to measure the fit of the data to the forward solution. A maximum and minimum value of the regularization parameter can be set by the user, but in a manner similar to the classical Levenburg-Marquard method for well-posed least squares problems the parameter can be varied within that range depending on the residual at each iteration.

Although it is common in inverse problems to think of (0.3.18) as a regularization scheme a more rational justification for the method is probabilistic. We consider the error in the measured data to be a sampled from a zero mean, possibly correlated, random variables. We then represent our a priori belief about the distribution of $\gamma$ as a probability distribution. The minimization (0.3.18) is the Maximum a posteriori (MAP) estimate for the case of independent Gaussian error and with prior distribution with log probability density proportional to $-\Psi(\gamma)$. A more sophisticated approach goes beyond Gauss distributions and MAP estimates and samples the posterior distribution using Markov Chain Monte Carlo methods [70] (see chapter 36 statistical inverse problems). As this involves a large number of forward problem solutions this is infeasible for large 
scale three dimensional EIT problems. However as computers increase in speed and memory size relative to price, we expect this will eventually become a feasible approach. It will make it easier to approach EIT with a specific question such "as what is the volume of the region with a specified conductivity" with the answer expressed as an estimate of the probability distribution. Going back to $(0.3 .18)$ the regularization parameter $\alpha^{2}$ controls the ratio of the variances of the prior and error distribution. In practice this choice of this parameter is somewhat subjective, and the usual techniques in choice of regularization parameter, and the caution in their application, are relevant.

Results of a geophysical ERT study are shown in fig 8 and we would like to thank the Geophysical Tomography Team, British Geological Survey (www.bgs.ac.uk/research/tomography) for this figure and the description of the survey we sumarize below. In this case ERT was used to identify the concentrations of leachate, the liquid that escapes from buried waste in a landfill site. The leachate can be extracted and recirculated to enhance the production of landfill gas, which can ultimately be used for electricity generation. It was important to use a non-invasive technique - the more standard practice of drilling exploratory wells could lead to new flow paths. Data were collected sequentially on 64 parallel survey lines, using a regular grid of electrode positions. The inter-line spacing was $15 \mathrm{~m}$ with a minimum electrode spacing of $5 \mathrm{~m}$ along line. For the current sources, electrode spacings between $15 \mathrm{~m}$ and $95 \mathrm{~m}$ were used, while electrode spacings for the measurement electrodes were between $5 \mathrm{~m}$ and $225 \mathrm{~m}$.

The inversion was performed using RES3DINV with the FE forward solver with a mesh generated using the measured surface topography. The two norm was used for both penalty term and error norm. Due to the large number of datum points (approx 85,000 in total), the dataset was split in four approximately equal volumes for subsequent inversion. The resulting resistivity models were then merged to produce a final model for the entire survey area. The resulting $3 \mathrm{D}$ resistivity model was used to identify a total of fourteen drilling locations for intrusive investigation. Eight wells were drilled and the results (i.e initial leachate strikes within these wells) were used to calibrate the resistivity model. Based on this ground-truth calibration a resistivity threshold value of $4 \Omega \mathrm{m}$ was used to represent the spatial distribution of leachate for volumetric analysis within the waste mass. A commercial vizualization package was used to display cross sections, iso-resistivity surfaces as well as topography and features on the surface and the boreholes. For other similar examples of geophysical ERT see [30] [31].

Experiments on tanks in process tomography or as simulated bodies for medical EIT show that several iterations of a non-linear method can improve the accuracy of conductivity and the shape of conductivity contours for known objects. In medical EIT it has yet to be demonstrated that the shape and electrode position can be measured and modelled with sufficient accuracy that the error in the linear approximation is greater than the modelling error. Although these technical difficulties are not, we hope, insurmountable. 


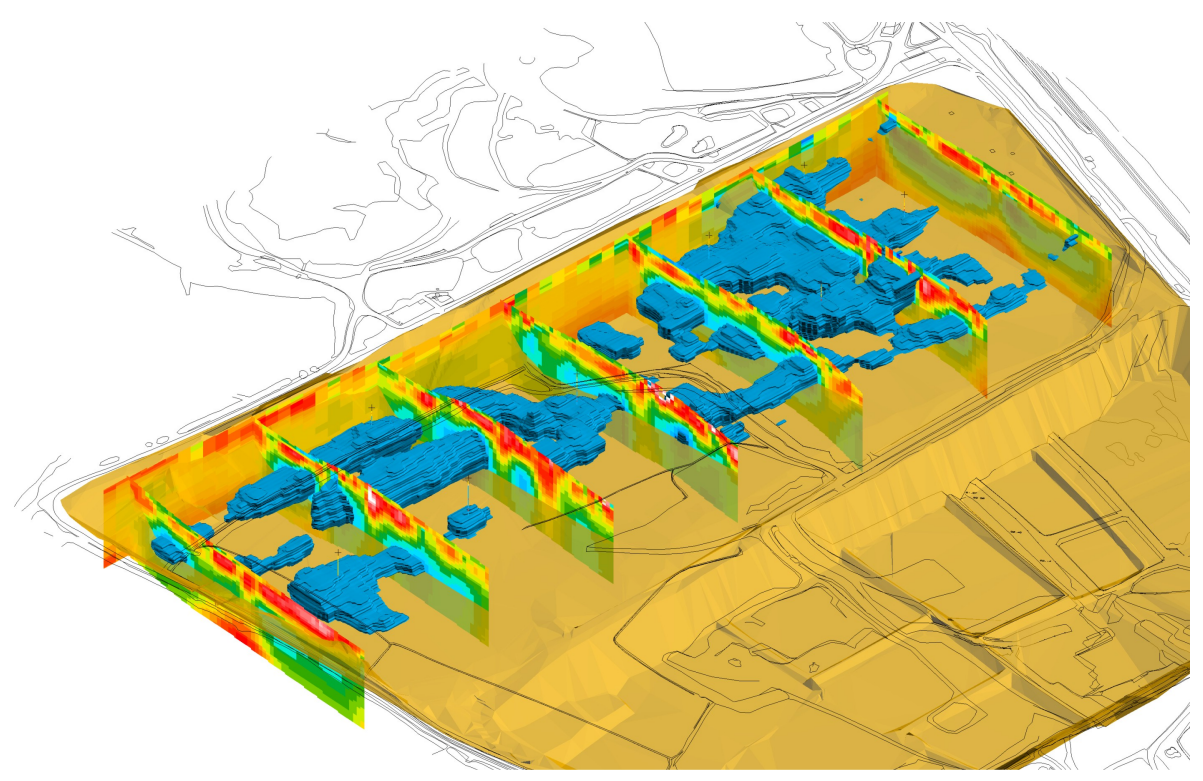

Figure 8: A three dimensional ERT survey of a commercial landfill site to map the volumetric distribution of leachate (opaque blue). Leachate is abstracted and Reproduction of any BGS materials does not amount to an endorsement by NERC or any of its employees of any product or service and no such endorsement should be stated or implied

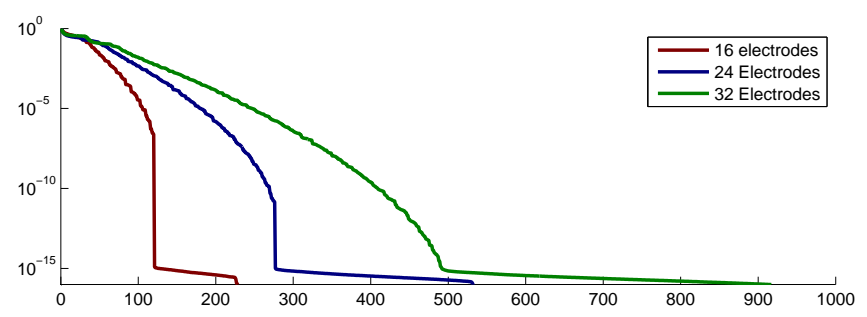

Figure 9: Normalised singular values of the Jacobian matrix from circular 2D model with $L=16,24$ and 32 electrodes. EIT measurements are made with trigonometric patterns such that the number of independent measurements from $L$ electrodes is $\frac{1}{2}(L-1) L$. Note the use of more degrees of freedom in the conductivity than the data so as to be able to study the effect of different numbers of electrodes using SVD 

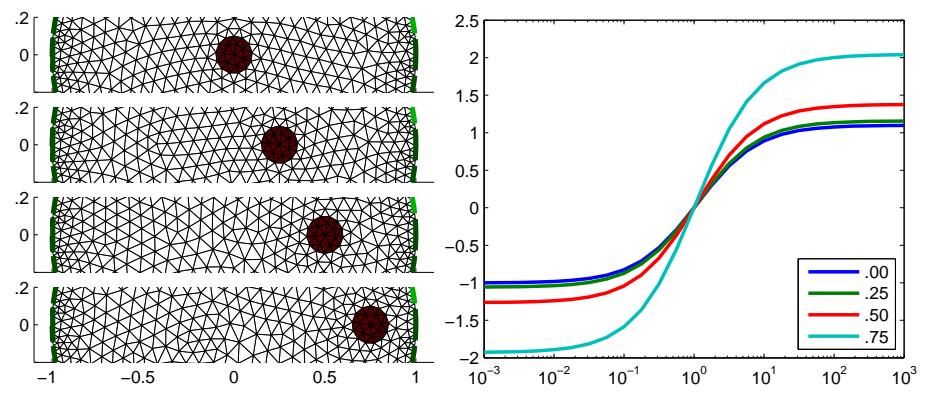

Figure 10: Saturation of EIT signals as a function of conductivity contrast. Left: Slices through a finite element model of a $2 \mathrm{D}$ circular medium with a circular conductivity target at four horizontal positions. EIT voltages are simulated at 32 electrodes for 31 trigonometric current patterns. Right: Right: Change in a voltage difference as a function of conductivity contrast (target vs. background) for each horizontal position (horizontal centre of contrast specified in legend). Vertical axis is normalised with respect to the maximum change from the central target, and scaled by the sign of conductivity change.

\subsubsection{Direct non-linear solution}

Nachman's [90, 91] (see also [96]) uniqueness result for the two dimensional case was essentially constructive and has resulted in a family of reconstruction algorithms called $\bar{\partial}$-methods or scattering transform methods. Nachman's method was implemented by Siltanen at al [102] in 2000. Of course there are few practical situations in which the two dimensional approximation is a good one - both the conductivity and the electrodes have to be translationally invariant. Flow in a pipe with long electrodes is one example in which it is a good approximation. We will sketch the main steps in the method (following Knudsen et al [78]) and refer the interested reader to the references for details.

We assume $\Omega$ is the unit disk for simplicity and we start with the Faddeev Green's function

$$
G_{k}(x):=\mathrm{e}^{\mathrm{i} k x} g_{k}(x), \quad g_{k}(x)=\frac{1}{(2 \pi)^{2}} \int_{\mathbb{R}^{2}} \frac{\mathrm{e}^{\mathrm{i} x \cdot \xi}}{|\xi|^{2}+2 k\left(\xi_{1}+\mathrm{i} \xi_{2}\right)} d \xi
$$

and the single layer potential

$$
\left(S_{k} \phi\right)(x):=\int_{\partial \Omega} G_{k}(x-y) \phi(y) d \theta(y) .
$$

Here $k=k_{1}+\mathrm{i} k_{2}$ and by abuse of notation we consider $x$ as a vector in $x \cdot \xi$ and a complex number $x_{1}+\mathrm{i} x_{2}$ in the complex product $k x$. By $\theta(y)$ we mean the angular polar coordinate of $y$. We assume we have the measured Dirichlet to Neumann map $\Lambda_{\gamma}$ and of course we know $\Lambda_{1}$. The first step in the algoritm 


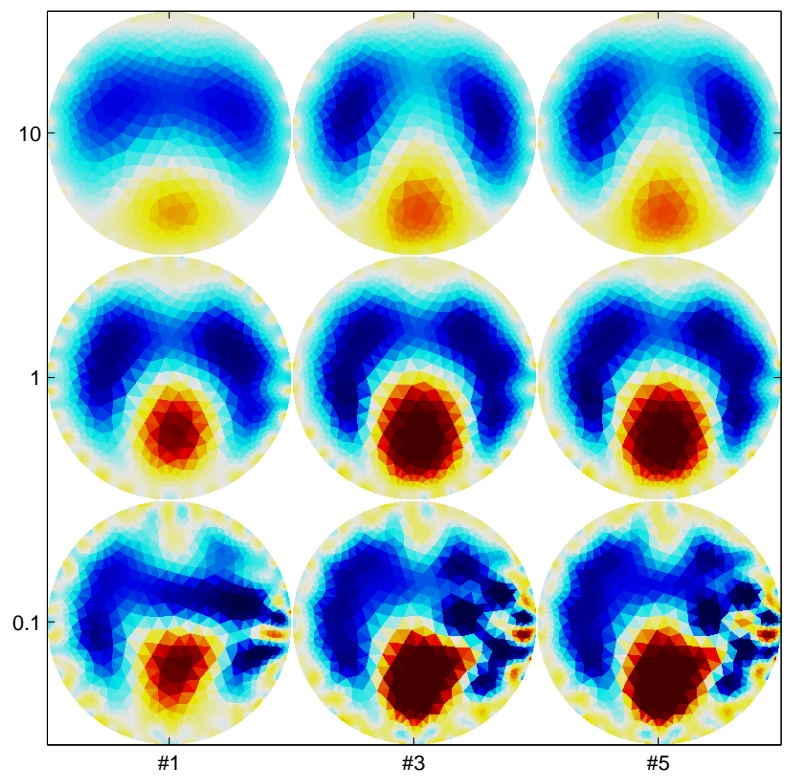

(a) Laplacian regularization

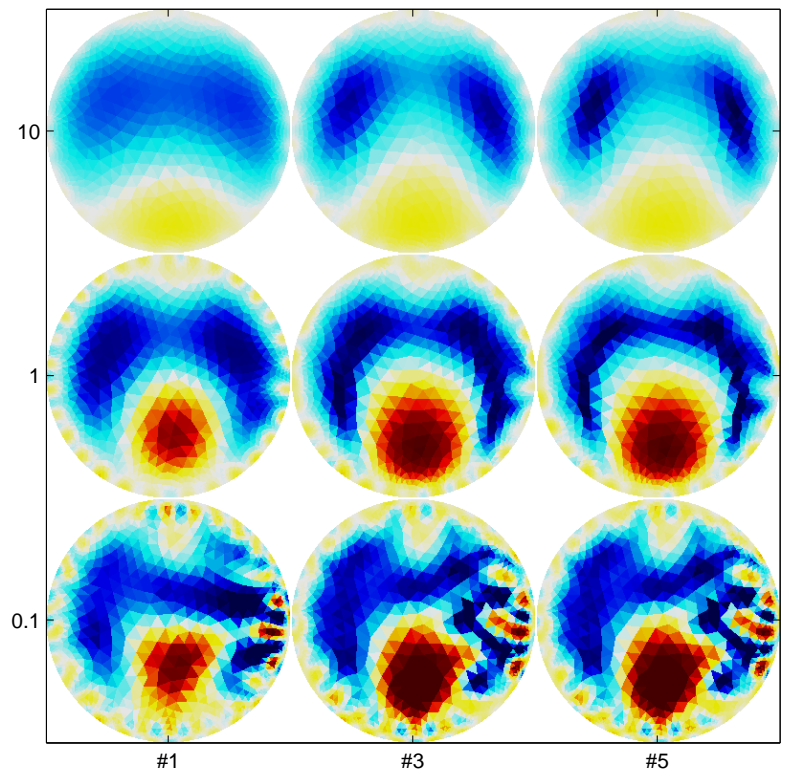

(b) Weighted diagonal regularization

Figure 11: Iteration (horizonal axis) and regularization parameter selection (vertial axis) for two choices of regularization matrix $\mathbf{R}$. Data are from the RPI chest phantom [66]. The regularization parameter $(\alpha$ in 0.3.19) in the middle row (1) was selected at the "knee" of the L-curve, indicating an appropritate level of regularization. Overregularization (top row) is shown for $10 \alpha$, and underregularization (bottom row) for $0.1 \alpha$. Columns indicate 1,3 or 5 iteration of (0.3.19). With increased iteration, we see improved separation of targets and more accurate conductivity estimates, although these improvements trade off against increased electrode artefacts due to model mismatch. The difference between the Laplacian and weighted diagonal regularization is shown in the increased smoothness of (a), especially in the underregularized case. 
is for each fixed $k$ to solve the linear Fredholm integral equation for a function $\psi(\cdot, k)$ on the boundary.

$$
\left.\psi(\cdot, k)\right|_{\partial \Omega}=\mathrm{e}^{i k x}-\left.S_{k}\left(\Lambda_{\gamma}-\Lambda_{1}\right) \psi(\cdot, k)\right|_{\partial \Omega}
$$

This is an explicit calculation of the Complex Geometrics Optics solution of Theorem 3. It is fed in to the calculation of what is called the non-physical scattering transform $\mathbf{t}: \mathbb{C} \rightarrow \mathbb{C}$ defined by

$$
\mathbf{t}(k)=\int_{\partial \Omega} \mathrm{e}^{\bar{k} \bar{x}}\left(\Lambda_{\gamma}-\Lambda_{1}\right) \psi(\cdot, k) d \theta
$$

Note here that $(0.3 .22)$ is a linear equation to solve the resulting $\psi$ depends nonlinearly on the data $\Lambda_{\gamma}$, and of course as $\psi$ depends on the data $\mathbf{t}$ is a nonlinear function of the data. The second step is to find the conductivity from the scattering data as follows. let $e_{x}(k):=\exp (\mathrm{i}(k x+\bar{k} \bar{x}))$. For each fixed $x$ we solve another integral equation

$$
V(x, k)=1+\frac{1}{(2 \pi)^{2}} \int_{\mathbb{R}^{2}} \frac{\mathbf{t}\left(k^{\prime}\right)}{\left(k-k^{\prime}\right) \bar{k}^{\prime}} e_{-x}\left(k^{\prime}\right) \overline{V\left(x, k^{\prime}\right)} d k_{1}^{\prime} d k_{2}^{\prime}
$$

finally setting $\gamma(x)=V(x, 0)^{2}$. The integral equation (0.3.24) is the solution to the partial differential equation

$$
\bar{\partial}_{k} V(x, k)=\frac{1}{4 \pi \bar{k}} \mathbf{t}(k) e_{-x}(k) \overline{V(x, k)}, \quad k \in \mathbb{C}
$$

where $\bar{\partial}_{k}=\partial / \partial \bar{k}$. Equation(0.3.25) is refered to as the $\bar{\partial}$ equation hence the name of the method.

The reconstruction procedure is therefore a direct nonlinear method in which the steps are the solution of linear equations. The only forward modelling required is the construction of $\Lambda_{1}$. In some practical realisations of this methods [66] an approximation to the scattering transform is used in which $\psi$ is replaced by an exponential

$$
\mathbf{t}^{\exp }(k)=\int_{\partial \Omega} \mathrm{e}^{\bar{k} \bar{x}}\left(\Lambda_{\gamma}-\Lambda_{1}\right) d \theta .
$$

In practical reconstruction schemes $\mathbf{t}$ or $\mathbf{t}^{\text {exp }}$ are replaced by an approximation truncated to zero for $|k|>R$ for some $R>0$, which effectiviely also truncates the domain of integration in (0.3.24) to the disk of radius $R$. Reconstruction of data from a two dimensional agar phantom simulating a chest was performed in [66] using truncated $\mathbf{t}^{\text {exp }}$, and in [67] a difference imaging version of the $\bar{\partial}$-method is implemented using a truncated scattering transform and applied to chest data. A rigorous regularization scheme for two dimensional $\bar{\partial}$-reconstruction is given in [78]. In this case the regularization is applied to the data, in a similar spirit to X-ray CT recontruction in which the data is filtered and then backprojected (see chapter 38 Tomography) and the regularization is 
applied in the filter on the data. In this sense it is harder to understand the regularized algorithm in terms of systematic a priori informationa applied to the image. As in CT this is traded off against having a fast explicit reconstruction algorithm that avoids iteration.

So far our discussion of $\bar{\partial}$-methods has been confined to two dimensional problems. At the time of writing three dimesnional direct reconstruction methods are in their infancy. A three dimensional $\bar{\partial}$-algorithm for small conductivities is outlined in [34] and it is yet to be seen if this will result in practical implementation with noisy data on a finite array of electrodes. See the thesis of Bikowski [16] for the latest steps in this direction. If these efforts are succesful, the impact on EIT is likely to be revolutionary.

\subsection{Conclusions}

Electrical impendance tomography and its relatives are among the most challenging inverse problems in imaging as the problem is non-linear and highly illposed. The problem has inspired detailed theoretical and numerical study and this has had an influence across a wide range of related inverse boundary value problems for (systems of) partial differential equations. Medical and industrial process applications have yet to realise their potential as routine methods while the equivalent methods in geophysics are well established. A family of direct non-linear solution techniques until recently only valid for the two dimensional problem, may soon be extended to practical three dimensional algorithms. If this happens fast three dimensional non-linear reconstruction may be possible on relatively modest computers. In some practical situations in medical and geophysical EIT the conductivity is anisotropic, in which case the solution is non-unique. A specification of the a priori information needed for a unique solution is poorly understood and practical reconstruction algorithms have yet to be proposed in the anisotropic case.

For a more complete summary of uniqueness results we refer the reader to the review article of Uhlmann [112]. For a review of biomedical applications of EIT we refer the reader to the recent book by Holder [61], while subsequent progress in the medical area can generally be found in special issues of the journal Physiological Measurement arising from the annual conferences on Biomedical Applications of EIT. A good reference for details of geophysical EIT reconstruction can be found in the manual[46] and the notes by Loke[84]. For applications in process tomography see [119] and the proceedings of the biennial World Congress on Industrial Process Tomography (www.isipt.org/wcipt) 


\section{Bibliography}

[1] A. Adler and W.R.B. Lionheart, Uses and abuses of EIDORS: An extensible software base for EIT, Physiol Meas 27, S25-S42, (2006).

[2] G. Alessandrini, Stable determination of conductivity by boundary measurements, Appl. Anal., Vol.27, (1988), 153-172.

[3] G. Alessandrini, Singular Solutions of Elliptic Equations and the Determination of Conductivity by Boundary Measurements, J. Differential Equations, Vol.84, No.2 (1990), 252-272.

[4] G. Alessandrini, Determining conductivity by boundary measurements, the stability issue., In: Applied and Industrial Mathematics, R. Spigler (ed.). Kluwer (1991), 317-324.

[5] G. Alessandrini, Open issues of stability for the inverse conductivity problem, J. Inverse Ill-Posed Problems, Vol.15, (2007), 451-460.

[6] G. Alessandrini and R. Gaburro, Determining Conductivity with Special Anisotropy by Boundary Measurements, SIAM J. MATH. ANAL., Vol.33 (2001), 153-171.

[7] G. Alessandrini and R. Gaburro, The local Calderón problem and the determination at the boundary of the conductivity., Commun. PDE, Vol.34 (2009), 918-936.

[8] G. Alessandrini and S. Vessella, Lipschitz stability for the inverse conductivity problem, Adv. Appl. Math., Vol.35 (2005), 207-241.

[9] H. Ammari, A. Buffa, J.-C Nédélec, A justification of eddy currents model for the Maxwell equations SIAM Journal on Applied Mathematics, 60, $1805-1823,(2000)$

[10] N. Aronszajn, A unique continuation theorem for solutions of elliptic partial differential equations or inequalities of second order, J. Math. Pures Appl., Vol.36, (1957), 235-249.

[11] K. Astala and L. Päivärinta, Calderón's inverse conductivity problem in the plane, Annals of Mathematics, 163, (2006), 265-299 
[12] D. Barber and B. Brown, Recent developments in applied potential tomography - APT, Information Processing in Medical Imaging, ed S L Bacharach (Amsterdam: Nijhoff) 10621, (1986)

[13] J. A. Barceló, D. Faraco and A. Ruiz, Stability of the inverse problem in the plane for less regular conductivities, J. Differential Equations., Vol.173 (2001), 231-270.

[14] J. A. Barceló, T. Barceló and A. Ruiz, Stability of Calderón Inverse Conductivity Problem in the Plane, J. Math. Pures Appl., Vol.88 (2007), 522556.

[15] C.A. Berenstein and E. Casadio Tarabusi, Integral geometry in hyperbolic spaces and electrical impedance tomography, SIAM J. Appl. Math. 56 75564, (1996)

[16] J. Bikowski, Electrical Impedance Tomography Reconstructions in Two and Three Dimensions; from Calderón to Direct Methods, Ph.D. thesis, Colorado State University, Fort Collins, CO, (2009).

[17] L. Borcea Electrical impedance tomography Inverse Problems 18 R99136, (2002) Borcea L Addendum to 'Electrical impedance tomography Inverse Problems 199978 (2003)

[18] A Borsic, B.M. Graham, A Adler, W.R.B. Lionheart, Total Variation Regularization in Electrical Impedance Tomography, IEEE TMI, 29, 1, 44$54,(2010)$

[19] E. Beretta and E. Francini, Lipschitz stability for the electrical impedance tomography problem: the complex case, Comm. in PDEs, Vol.36 (2011), 17231749 .

[20] E. Beretta, M. V. de Hoop and L. Qiu, Lipschitz Stability of an Inverse Boundary Value Problem for a Schrdinger-Type Equation, SIAM J. Math. Analysis Vol.45, No.2 (2013), 679-699.

[21] R. Brown, Recovering the conductivity at the boundary from the Dirichlet to Neumann map: a pointwise result, J. Inverse Ill-Posed Problems, Vol.9 (2001), 567-574.

[22] R. Beals and R. Coifman, Transformation spectrales et equation d'evolution non lineares, Seminaire Goulaouic-Meyer-Schwarz, exp.9, 1981-1982, (1982)

[23] R. Beals and R.R. Coifman. Linear spectral problems, non-linear equations and the $\bar{\partial}$-method, Inverse Problems, 5:87130, (1989).

[24] R. Brown and R. Torres, Uniqueness in the inverse conductivity problem for conductivities with $3 / 2$ derivatives in $L^{p}, p>2 n$, J. Fourier Anal. Appl., Vol.9 (2003), 1049-56. 
[25] R. Brown, Global Uniqueness in the Impedance-Imaging Problem for Less Regural Conductivities, SIAM J. Math. Anal., Vol.27, no. 4 (1996), 1049.

[26] R. Brown and G. Uhlmann, Uniqueness in the inverse conductivity problem with less regular conductivities in two dimensions, Commun. PDE., Vol.22 (1997), 1009-1027.

[27] A.L. Bukhgeim and G. Uhlmann, Recovery a potential from partial Cauchy data., Commun. PDE., Vol.27 (2002), 653-668.

[28] A. P. Calderón, On an inverse boundary value problem, Seminar on Numerical Analysis and its Applications to Continuum Physics (Rio de Janeiro, 1980), Rio de Janeiro: Soc. Brasil. Mat., 65-73.

[29] A. P. Calderón, On an inverse boundary value problem, Comput. Appl. Math., Vol.25, No2-3 (2006), 133-138. (Note this reprint has some different typographical errors from the original: in particular on the first page the Dirichlet data for $w$ is $\phi$ not zero).

[30] J.E. Chambers, P.I. Meldrum, R.D. Ogilvy and P.B. Wilkinson Characterisation of a NAPL-contaminated former quarry site using electrical impedance tomography, Near Surface Geophysics, 79-90, (2005)

[31] J.E. Chambers, O. Kuras, P.I. Meldrum, R. D. Ogilvy, and J. Hollands, Electrical resistivity tomography applied to geologic, hydrogeologic, and engineering investigations at a former waste-disposal site, Geophysics, 71, B231B239, (2006)

[32] K. Cheng, D. Isaacson, J.C. Newell and D.G. Gisser, Electrode models for electric current computed tomography, IEEE Trans. on Biomedical Engineering 36 918-24, (1989)

[33] M. Cheney, D. Isaacson and J.C. Newell. Electrical Impedance Tomography. SIAM Review. 41, 85-101, (1999).

[34] H. Cornean, K. Knudsen, S. Siltanen, Towards a D-bar reconstruction method for three dimensional EIT J. Inverse Ill-posed Probl. 14, 111134 (2006)

[35] Y Colin de Verdière, I. Gitler, D. Vertigan, Réseaux électriques planaires. II. Comment. Math. Helv., 71, 144-167 (1996)

[36] M. Di Cristo, Stable determination of an inhomogeneous inclusion by local boundary measurements, J. Computational and Applies Mathematics, Vol.198 (2007), 414-425.

[37] S. Ciulli, S. Ispas, and M. K. Pidcock, Anomalous thresholds and edge singularities in electrical impedance tomography, J. Math. Phys. 37, 4388 (1996) 
[38] D.C. Dobson, Stability and regularity of an inverse elliptic boundary value problem, Technical Report TR90-14 Rice University, Dept of Math. Sciences, (1990)

[39] B.H. Doerstling A 3-d reconstruction algorithm for the linearized inverse boundary value problem for Maxwell's equations $\mathrm{PhD}$ thesis, Rensselaer Polytechnic Institute, (1995)

[40] V. Druskin, The unique solution of the inverse problem of electrical surveying and electrical well-logging for piecewise-constant conductivity, Izv. earth Phys. Vol. 18 (1982), 51-53 (in Russian).

[41] V. Druskin, On uniqueness of the determination of the three-dimensional underground structures from surface measurements with variously positioned steady-state or monochromatic field sources, Sov. Phys.-Solid Earth Vol. 21 (1985), 210-214 (in Russian).

[42] V. Druskin, On the uniqueness of inverse problems for incomplete boundary data, SIAM J. Appl. Math. Vol. 58, No.5 (1998), 1591-1603.

[43] R. Gaburro, Sul Problema Inverso della Tomografia da Impedenza Elettrica nel Caso di Conduttivitá Anisotropa, Tesi di Laurea in Matematica, Universitá degli Studi di Trieste, 1999.

[44] R. Gaburro, Anisotropic Conductivity. Inverse Boundary Value Problems, PhD Thesis, University of Manchester Institute of Science and Technology (UMIST), Manchester, 2003.

[45] R. Gaburro and W.R.B. Lionheart, Recovering Riemannian metrics in monotone families from boundary data., Inverse Problems, Vol.25: 045004 (2009), (14pp).

[46] Geotomo Software, RES3DINV ver. 2.16, Rapid 3D Resistivity and IP inversion using the least-squares method, Geotomo Software, Malaysia. www.geoelectrical.com, October 2009

[47] D.G. Gisser, D. Isaacson and J.C. Newell. Electric current computed tomography and eigenvalues. SIAM J. Appl. Math. 50:1623-1634, (1990).

[48] H. Griffiths and J. Jossinet, Bioelectric tissue spectroscopy from multifrequency EIT, Physiol. Meas., Suppl. 2A, 15, 2935, (1994).

[49] H. Griffiths, Magnetic induction tomography, Meas. Sci. Technol. 12 11261131 (2001)

[50] B. Haberman and D. Tataru, Uniqueness in Calderòn problem with Lipschitz conductivities, Duke Math J., Vol.162, No. 3 (2013), 435-625.

[51] M. Hanke, On real-time algorithms for the location search of discontinuous conductivities with one measurement, Inverse Problems 24 045005, (2008) 
[52] M Hanke, B Harrach, and N Hynöven, Justification of point electrode models in electrical impedance tomography, Math. Models Methods Appl. Sci. 21, 1395 (2011)

[53] M Hanke, B Schappel, The Factorization Method for Electrical Impedance Tomography in the Half-Space SIAM J. Appl. Math. 68, 907-924 (2008)

[54] P. Hähner, A periodic Faddeev-type solution operator, J. Diff. Eqns, Vol.128, 300-308, (1996).

[55] B Harrach and JK Seo, Exact shape-reconstruction by one-step linearization in electrical impedance tomography, SIAM J. Math. Anal. 42, 15051518, (2010)

[56] S.M. Huang, A. Plaskowski, C.G. Xie and M.S. Beck, Capacitance-based tomographic flow imaging system, Electronics Letters, 24, 418-419, (1988)

[57] H. Heck and J.-N. Wang, Stability estimates for the inverse boundary value problem by partial Cauchy data., Inverse Problems, Vol.22 (2006), 17871796.

[58] LM Heikkinen, TVilhunen, RM West and M. Vauhkonen, Simultaneous reconstruction of electrode contact impedances and internal electrical properties: II. Laboratory experiments, Meas. Sci. Technol. 13 p1855 (2002)

[59] S. Heinrich, H. Schiffmann, A. Frerichs, A. Klockgether-Radke, I. Frerichs, Body and head position effects on regional lung ventilation in infants: an electrical impedance tomography study, Intensive Care Med., 32:1392-1398, 2006.

[60] R.P. Henderson, J.G. Webster, An Impedance Camera for Spatially Specific Measurements of the Thorax, Biomedical Engineering, IEEE Transactions on , vol.BME-25, no.3, pp.250-254, (1978)

[61] D.S. Holder Electrical Impedance Tomography methods History and Applications, Institute of Physics Publishing, Bristol, 2005.

[62] M. Ikehata, The enclosure method and its applications, Chapter 7 in "Analytic extension formulas and their applications" (Fukuoka, 1999/Kyoto, 2000), Int. Soc. Anal. Appl. Comput., Kluwer Acad. Pub., 9, 87-103, (2001)

[63] M. Ikehata and S. Siltanen, Numerical method for nding the convex hull of an inclusion in conductivity from boundary measurements, Inverse Problems, 16, 273-296, (2000)

[64] D. Ingerman, J.A. Morrow, On a characterization of the kernel of the Dirichlet-to-Neumann map for a planar region, SIAM J. Math. Anal, 29, 106115, (1998) 
[65] D. Isaacson, Distinguishability of conductivities by electric current computed tomography,IEEE Trans. on Medical Imaging 5, 92-95, (1986)

[66] D. Isaacson, J.L. Mueller, J. Newell and S. Siltanen, Reconstructions of chest phantoms by the d-bar method for electrical impedance tomography, IEEE Transactions on Medical Imaging 23, 821- 828 (2004)

[67] D. Isaacson D, J.L. Mueller, J. Newell and S. Siltanen , Imaging Cardiac Activity by the D-bar Method for Electrical Impedance Tomography. Physiological Measurement 27, S43-S50, (2006)

[68] V. Isakov, Completeness of products of solutions and some inverse roblems for PDE, J. Diff Eqns, Vol.92 (1991), 305-317.

[69] V. Isakov, On the uniqueness in the inverse conductivity problem with local data., Inverse Problems and Imaging, Vol.1 (2007), 95-105.

[70] J. Kaipio, V. Kolehmainen, E. Somersalo and M. Vauhkonen, Statistical inversion and Monte Carlo sampling methods in electrical impedance tomography, Inverse Problems 16 1487-1522, (2000)

[71] H. Kang and K. Yun, Boundary determination of conductivities and Riemannian metrics via local Dirichlet-to-Neumann operator, SIAM J. Math. Anal. , Vol.34 (2002), 719-735.

[72] C. Kenig and M. Salo Recent progress in the Calderòn problem with partial data, to appear in Contemp. Math.

[73] C. Kenig and M. Salo The Calderòn problem with partial data on manifolds and applications, to appear in Analysis \& PDE.

[74] C. Kenig, J. Sjöstrand and G. Uhlmann, The Calderón problem with partial data.,Ann. Math., Vol.165 (2007), 567-591.

[75] Y. Kim and H.W. Woo A prototype system and reconstruction algorithms for electrical impedance technique in medical body imaging Clin. Phys. Physiol. Meas. 8 63-70, (1987)

[76] R. Kohn and M. Vogelius, Identification of an Unknown Conductivity by Means of Measurements at the Boundary, SIAM-AMS Proc., Vol.14 (1984), 113-123.

[77] R. Kohn and M. Vogelius, Determining Conductivity by Boundary Measurements II. Interior Results, Comm. Pure Appl. Math., Vol.38 (1985), 643-667.

[78] K. Knudsen, M. Lassas, J.L. Mueller and S. Siltanen, Regularized D-bar method for the inverse conductivity problem Inverse Problems and Imaging 3. 599-62, (2009) 
[79] M. Lassas and G. Uhlmann, Determining a Riemannian manifold from boundary measurements, Ann. Sci. École Norm. Sup., Vol.34 (2001), 771787 .

[80] M. Lassas, M. Taylor and G. Uhlmann, The Dirichlet-to-Neumann map for complete Riemannian manifolds with boundary, Commun. Geom. Anal., Vol.11 (2003), 207-222.

[81] W. R. B. Lionheart, Conformal Uniqueness Results in Anisotropic Electrical Impedance Imaging, Inverse Problems Vol.13 (1997), 125-134.

[82] J. M. Lee and G. Uhlmann, Determining anisotropic real-analytic conductivities by boundary measurements, Comm. Pure Appl. Math., Vol.42 (1989), 1097-112.

[83] L. Liu, Stability Estimates for the Two-Deimensional Inverse Conductivity Problem, PhD Thesis, University of Rochester, New York, 1997.

[84] M.H. Loke, Tutorial: 2-D and 3-D electrical imaging surveys, Geotomo Software, www.geoelectrical.com, 2010

[85] M.H. Loke and R.D. Barker, Rapid least- squares inversion by a quasiNewton method, Geophysical Prospecting, 44, 131152, (1996)

[86] M.H. Loke, J.E. Chambers and R.D. Ogilvy, Inversion of $2 D$ spectral induced polarization imaging data, Geophysical Prospecting, 54, 287 - 301, (2006)

[87] N. Mandache, Exponential instability in an inverse problem for the Schrödinger equation., Inverse Problems, Vol.17 (2001), 1435-1444.

[88] N. G. Meyers, An $L^{p}$ estimate for the gradient of solutions of second order elliptic divergence equations, Ann. Scuola Norm. Sup-Pisa, Vol.17, No.3, 189-206. (1963)

[89] M. Molinari, B.H. Blott,S.J. Cox, and G.J. Daniell, Optimal imaging with adaptive mesh refinement in electrical tomography, Physiological Measurement, 23, (1), 121-128, (2002)

[90] A. Nachman, Reconstructions from boundary measurements, Ann. Math., Vol.128 (1988), 531-576.

[91] A. Nachman, Global Uniqueness for a two Dimensional Inverse Boundary Value Problem, Ann. Math., Vol.143 (1996), 71-96.

[92] A. Nachman, J. Sylvester and G. Uhlmann, An n-dimensional BorgLevinson theorem., Comm. Math. Phys., Vol.115 (1988), 593-605.

[93] G. Nakamura and K. Tanuma, Local determination of conductivity at the boundary from the Dirichlet-to-Neumann map.,Inverse Problems, Vol.17 (2001), 405-419. 
[94] G. Nakamura and K. Tanuma, Direct determination of the derivatives of conductivity at the boundary from the localized Dirichlet to Neumann map, Comm. Korean Math. Soc., Vol.16 (2001), 415-425.

[95] G. Nakamura and K. Tanuma, Formulas for reconstrucing conductivity and its normal derivative at the boundary from the localized Dirichlet to Neumann map, In: Hon, Y.-C., Yamamoto, M., Cheng, J., Lee,J.-Y., eds. Proceeding, International Conference on Inverse Problem-Recent Development in Theories and Numerics. River Edge, NY: Would Scientific, 192-201.

[96] Novikov, R. G. (1988) A multidimensional inverse spectral problem for the equation $-\Delta \psi+(v(x)-E u(x)) \psi=0$. (Russian) Funktsional. Anal. i Prilozhen. 22, no. 4, 11-22, 96; translation in Funct. Anal. Appl. 22 (1988), no. 4, 263-272 (1989))

[97] K, Paulson, W. Breckon, M. Pidcock, Electrode Modeling In ElectricalImpedance Tomography SIAM J Appl Math, 1992, 52, pp.1012-1022

[98] L. Päivärinta, A. Panchenko and G. Uhlmann, Complex geometrical optics solutions for Lipschitz conductivities, Rev. Mat. Iberoamericana, Vol.19 (2003), 57-72.

[99] N Polydorides, W.R.B. Lionheart, A Matlab toolkit for three-dimensional electrical impedance tomography: a contribution to the Electrical Impedance and Diffuse Optical Reconstruction Software project, Meas. Sci. Technol. 13, 1871-1883, (2002)

[100] A.D. Seagar, Probing with low frequency electric current, PhD Thesis, University of Canterbury, Christchurch, NZ, (1983).

[101] A. D. Seagar and R. H. T. Bates, Full-wave computed tomography. Pt 4: Low-frequency electric current CT, Inst. Electr. Eng Proc. Pt. A, vol. 132, pp. 455-466, (1985).

[102] S. Siltanen, J.L. Mueller and D. Isaacson, An implementation of the reconstruction algorithm of $A$. Nachman for the 2-D inverse conductivity problem, Inverse Problems 16 68199, (2000)

[103] M Soleimani, W.R.B. Lionheart, 2005. Nonlinear image reconstruction for electrical capacitance tomography experimental data using. Measurement Science \& Technology, 16 (10), pp. 1987-1996.

[104] M. Soleimani, W.R.B. Lionheart, O. Dorn Level set reconstruction of conductivity and permittivity from boundary electrical measurements using expeimental data, Inverse Problems in Science and Engineering,14, 193$210,(2006)$. 
[105] E. Somersalo, M. Cheney and D. Isaacson ,Existence and uniqueness for electrode models for electric current computed tomography. SIAM J. Appl. Math., 52, 1023-1040, (1992)

[106] N.K. Soni, Breast Imaging using Electrical Impedance Tomography, PhD thesis, Dartmouth College, NH, (2005)

[107] J. Sylvester, An anisotropic inverse boundary value problem, Comm. Pure. Appl. Math., Vol.43 (1990), 201-232.

[108] J. Sylvester and G. Uhlmann, A uniqueness theorem for an inverse boundary value problem in electrical prospection, Commun. Pure Appl. Math., Vol.39 (1986), 92-112.

[109] J. Sylvester and G. Uhlmann, A Global Uniqueness Theorem for an Inverse Boundary Valued Problem, Ann. Math., Vol.125 (1987), 153-169.

[110] J. Sylvester and G. Uhlmann, Inverse boundary value problems at the boundary - continuous dependence., Commun. Pure Appl. Math., Vol.41 (1988), 197-221.

[111] A. Tamburrino and G. Rubinacci, A new non-iterative inversion method for electrical resistance tomography, Inverse Problems, 18, 1809-1829, (2002)

[112] G. Uhlmann, Topical review: Electrical impedance tomography and Calderón's problem, Inverse Problems, Vol.25 , 123011 (39pp), (2009)

[113] M. Vauhkonen, W.R.B. Lionheart, L.M. Heikkinen, P.J. Vauhkonen, and J.P. Kaipio, A MATLAB package for the EIDORS project to reconstruct two-dimensional EIT images. Physiological Measurement, 22, 107111. (2001)

[114] M. Vauhkonen, Electrical Impedance Tomography and Prior Information, PhD Thesis, University of Kuopio (1997)

[115] M. Vauhkonen, P.A. Karjalainen, and J.P. Kaipio, A Kalman Filter approach to track fast impedance changes in electrical impedance tomography, IEEE Trans Biomed Eng, 45, 486-493, (1998)

[116] R.M. West, M. Soleimani, R.G. Aykroyd, and W.R.B. Lionheart, Speed Improvement of MCMC Image Reconstruction in Tomography by Partial Linearization, International Journal of Tomography \& Statistics, Vol. 4, No. S06, 13-23, (2006).

[117] R.M. West, X Jia, and R.A. Williams, Parametric modelling in industrial process tomography, Chemical Engineering Journal, 77, 31-36, (2000) 
[118] W.Q. Yang, D.M. Spink, T.A. York, and H. McCann, An imagereconstruction algorithm based on Landwebers iteration method for electrical-capacitance tomography, Measurement Science, and Technology, 10, 10651069, (1999)

[119] T. York Status of electrical tomography in industrial applications J. Electron. Imaging, 10, 608 (2001) 